\title{
Sharp Error Estimates \\ for a Finite Element-Penalty Approach to a Class of Regulator Problems*
}

\author{
By Goong Chen, Wendell H. Mills, Jr., ${ }^{* *}$ Shunhua Sun and David A. Yost
}

\begin{abstract}
Quadratic cost optimal controls can be solved by penalizing the governing linear differential equation [2], [9]. In this paper, we study the numerical analysis of this approach using finite elements. We formulate the geometric condition $(\mathrm{H})$ which requires that pairs of certain related finite-dimensional approximation spaces form "angles" which are bounded away from the " $180^{\circ}$ angle". Under condition $(\mathrm{H})$, we prove that the penalty parameter $\varepsilon$ and the discretization parameter $h$ are independent in the error bounds, thereby giving sharp asymptotic error estimates. This condition $(\mathrm{H})$ is shown to be also a necessary condition for such independence. Examples and numerical evidence are also provided.
\end{abstract}

0. Introduction. Consider the optimal control problem: Given the quadratic cost functional $J$,

$$
J(x, u) \equiv \int_{0}^{T}\left[\left\langle\dot{x}, N_{1} \dot{x}\right\rangle_{\mathbf{R}^{n}}+\left\langle x, N_{2} x\right\rangle_{\mathbf{R}^{n}}+\langle u, M u\rangle_{\mathbf{R}^{m}}\right] d t,
$$

solve

$$
\operatorname{Min}_{(x, u) \in H_{0 n}^{1} \times L_{m}^{2}} J(x, u)
$$

subject to

$$
\left\{\begin{array}{l}
\dot{x}(t)=A(t) x(t)+B(t) u(t)+f(t), \quad t \in[0, T], \\
x(0)=0,
\end{array}\right.
$$

where $x(t) \in \mathbf{R}^{n}$ is the state at time $t, u(t) \in \mathbf{R}^{m}$ is the control at $t, A(t)$ and $B(t)$ are, respectively, $n \times n$ and $n \times m$ time-varying matrices, and $f$ is the inhomogeneous forcing term.

In the cost functional $J$, we assume

$$
\left\{\begin{array}{r}
N_{1}, N_{2}, M \text { are constant } n \times n, n \times n, \text { and } m \times m \text { symmetric } \\
\text { positive semi-definite matrices, } \\
\left\langle x, N_{1} x\right\rangle_{\mathbf{R}^{n}} \geqslant \nu\|x\|_{\mathbf{R}^{n}}^{2},\langle u, M u\rangle_{\mathbf{R}^{m}} \geqslant \nu\|u\|_{\mathbf{R}^{m}}^{2} \text { for all } x \in \mathbf{R}^{n}, u \in \mathbf{R}^{m}, \\
\text { where } \nu>0, \text { is independent of } x \text { and } u .
\end{array}\right.
$$

The standard Sobolev norms and spaces used are as follows.

Received November 17, 1981; revised February 26, 1982 and May 3, 1982.

1980 Mathematics Subject Classification. Primary 34H05, 49D30, 65N30; Secondary 41A65.

* Supported in part by NSF Grant MCS 81-01892. Work completed while the third author was visiting Purdue University.

** Current address: Research and Development Department, Standard Oil of Ohio, Cleveland, Ohio 44128 . 


$$
\begin{gathered}
\|y\|_{H_{l}^{k}}^{2} \equiv \sum_{j=0}^{k} \int_{0}^{T}\left\|y^{(j)}(t)\right\|_{\mathbf{R}^{\prime}}^{2} d t, \\
H_{l}^{k} \equiv H_{l}^{k}(0, T) \equiv\left\{y:[0, T] \rightarrow \mathbf{R}^{l} \mid y^{(i)}\right. \text { is absolutely continuous, } \\
H_{0 l}^{1} \equiv\left\{\begin{array}{c}
\left.y \in H_{l}^{1} \mid y(0)=0\right\}, \quad\|y\|_{H_{0 l}^{\prime}} \equiv\|y\|_{H_{l}^{\prime}}, \\
L_{l}^{2} \equiv L_{l}^{2}(0, T) \equiv H_{l}^{0}(0, T),
\end{array}\right.
\end{gathered}
$$

for $l \in \mathbf{Z}^{+} \equiv\{1,2,3, \ldots\}$ and $k \in \mathbf{N} \equiv\{0,1,2, \ldots\}$.

Using calculus of variations, $(0.1)$ and $(0.2)$ are equivalent to the variational formulation

$$
a\left(\left[\begin{array}{l}
x \\
u
\end{array}\right],\left[\begin{array}{l}
y \\
v
\end{array}\right]\right)=0 \quad \text { for all }\left[\begin{array}{l}
y \\
v
\end{array}\right] \in H_{0 n}^{1} \times L_{m}^{2}, \dot{y}=A y+B v, y(0)=0
$$

where

$$
a\left(\left[\begin{array}{l}
x \\
u
\end{array}\right],\left[\begin{array}{l}
y \\
v
\end{array}\right]\right) \equiv \int_{0}^{T}\left[\left\langle\dot{x}, N_{1} \dot{y}\right\rangle_{\mathbf{R}^{n}}+\left\langle x, N_{2} y\right\rangle_{\mathbf{R}^{n}}+\langle u, M v\rangle_{\mathbf{R}^{m}}\right] d t
$$

A feasible approach for computing the optimal control $\hat{u}$ and the corresponding optimal state $\hat{x}$ is by penalizing the governing equation $(0.2)$ : we solve the unconstrained problem

$$
\operatorname{Min}_{(x, u) \in H_{0 n}^{1} \times L_{m}^{2}} J_{\varepsilon}(x, u, f) \equiv J(x, u)+\frac{1}{\varepsilon}\|\dot{x}-A x-B u-f\|_{L_{n}^{2}}^{2}, \quad \varepsilon>0,
$$

and let $\varepsilon$ tend to zero to obtain convergence. This approach was first introduced by A. V. Balakrishnan [2] and J. L. Lions [9].

Note that the form of the cost functional $J$ requires that the (weighted) rate of change of the state $\dot{x}$ be minimized, in addition to both the (weighted) state $x$ and control $u$. This is an important technical assumption in our paper. We also note that an inhomogeneous initial condition $x(0)=x_{0}$ can be reduced to the zero initial condition (as in (0.2)) by the change of variable $y(t)=x(t)-x_{0}$.

From the Poincaré inequality, the expressions

$$
\int_{0}^{T}\left\langle\dot{x}(t), N_{1} \dot{x}(t)\right\rangle d t, \quad \int_{0}^{T}\left[\left\langle\dot{x}(t), N_{1} \dot{x}(t)\right\rangle+\left\langle x(t), N_{2} x(t)\right\rangle\right] d t
$$

in $J$ define equivalent norms in the Hilbert space $H_{0 n}^{1}$. We assume $A(t)$ is sufficiently smooth such that, for $y \in H_{0 n}^{1}$,

(0.6) $\|\dot{y}-A y\|_{L_{n}^{2}}=\left(\int_{0}^{T}|\dot{y}(t)-A(t) y(t)|^{2} d t\right)^{1 / 2} \geqslant c\|y\|_{H_{n}^{1}}$ for some $c>0$

(i.e., the above defines an equivalent norm in $H_{0 n}^{1}$ ). It is trivial to see that this holds when $A$ is a constant matrix.

Let $S_{h_{i}}^{1} \subset H_{0 n}^{1}$ and $S_{h_{i}}^{2} \subset L_{m}^{2}$ be two sequences of increasing finite-dimensional spaces such that

$$
\lim _{h_{i} \downarrow 0} \inf _{y \in S_{h_{i}}^{1}}\|x-y\|_{H_{0 n}^{1}}=0, \quad \lim _{h_{i} \downarrow 0} \inf _{v \in S_{h_{i}}^{2}}\|u-v\|_{L_{m}^{2}}=0 \quad \forall(x, u) \in H_{0 n}^{1} \times L_{m}^{2} .
$$


The finite element approximation is to minimize (0.5) over $S_{h}^{1} \times S_{h}^{2} \subset H_{0 n}^{1} \times L_{m}^{2}$ ( $h=h_{i}$ for some $i$ ). Let $\left(\hat{x}_{h}^{\varepsilon}, \hat{u}_{h}^{\varepsilon}\right)$ be the unique minimizer of $(0.5)$ in $S_{h}^{1} \times S_{h}^{2}$. Our goal is to analyze the error

$$
\left\|\hat{x}_{h}^{\varepsilon}-\hat{x}\right\|_{H_{0 n}^{1}}+\left\|\hat{u}_{h}^{\varepsilon}-\hat{u}\right\|_{L_{m}^{2}}
$$

with respect to the penalty parameter $\varepsilon$ and the discretization parameter $h$.

Numerical analysis of penalty problems of similar nature has been studied in [6], [7], for example. In those works, the penalty parameter $\varepsilon$ is often found to be coupled with (or dependent upon) $h$. In [4], Chen and Mills give some sharp estimates for a primal-penalty-finite element computation scheme which show that, in the error bounds for that problem and approach, $\varepsilon$ and $h$ are actually independent of each other. The problem in [4] is simpler than the one being studied here because the penalized constraint is finite-dimensional. As we will see later on, for the problem and approach considered here, the independence of $\varepsilon$ and $h$ will not hold in general.

The main result of our paper is as follows. We show that sharp estimates (cf. (3.4)) hold if and only if the approximating finite-dimensional spaces $\left\{\left(S_{h_{i}}^{1}, S_{h_{i}}^{2}\right)\right\}_{i=1}^{\infty}$ satisfy a certain special property, namely, Condition $(\mathrm{H})$ in Section 3. This condition requires that pairs of certain related finite-dimensional subspaces form "angles" which are bounded away from the " $180^{\circ}$ angle".

In Section 1, we introduce some basic facts about penalty and study the wellposedness of exact solutions and penalized solutions with respect to the inhomogeneous data $f$. The relations between the solutions and the inhomogeneous data $f$ are linear and expressed in terms of certain linear operators $\mathcal{L}, \mathcal{E}_{\varepsilon}, \mathcal{L}_{h}$ and $\mathcal{L}_{h} \varepsilon$. Basic errors between the exact (or, the discretized) solution and the penalized solution are estimated.

It is found in this paper that the validity of sharp error bounds can be studied in terms of an abstract approximation problem. This problem has considerable theoretical interest in its own right and is thoroughly examined in Section 2. Necessary and sufficient conditions are formulated for the solvability of this problem

In Section 3, we give the main estimates in Theorem 12. Condition $(\mathrm{H})$, which is obtained through the study of the abstract approximation problem in Section 2, is seen to be necessary and sufficient for Theorem 12 to hold. Error bounds in the case without $(\mathrm{H})$ are given in Theorem 20.

In Section 4, we apply the theory to some examples. Numerical computations obtain suggest that the errors indicated in Theorem 12 are sharp.

As with the penalty method, the stiffness matrix (associated with the quadratic form $J_{\varepsilon}$ in (0.5)) usually has a large condition number, thereby producing considerable numerical instability. This instability can be circumvented by using the standard matrix iterative refinement technique. Numerical results indicate that the penalty method is quite accurate and efficient when compared with other methods, e.g., the primal [4] or the dual [3] methods.

In the design of optimal regulators, the matrix $N_{1}$ appearing in $J$ is usually 0 [11]. In this situation, computationally, the penalty method also produces accurate results. Nevertheless, at this stage, no satisfactory error estimates like (3.4) for such problems are available. It remains a challenging research work yet to be completed by control theorists and numerical analysts. 
1. Finite Element Approximations of the Penalized Problem. We consider the unconstrained penalized problem

$$
\underset{(x, u) \in H_{0 n}^{1} \times L_{m}^{2}}{\operatorname{Min}} J_{\varepsilon}(x, u, f),
$$

where

$$
\begin{aligned}
J_{\varepsilon}(x, u, f) \equiv & \left\langle\dot{x}, N_{1} \dot{x}\right\rangle_{L_{n}^{2}}+\left\langle x, N_{2} x\right\rangle_{L_{n}^{2}}+\langle u, M u\rangle_{L_{m}^{2}} \\
& +\frac{1}{\varepsilon}\|\dot{x}-A x-B u-f\|_{L_{n}^{2}}^{2}, \quad \varepsilon>0,
\end{aligned}
$$

and (0.3), (0.6) hold.

Let $\left(\hat{x}_{\varepsilon}, \hat{u}_{\varepsilon}\right)$ be the unique minimizer of $(1.1)$ and let $(\hat{x}, \hat{u})$ be the optimal state and control of problem (0.1) and (0.2). From the work of Polyak [10], we see that

$$
\begin{aligned}
\left\|\hat{x}_{\varepsilon}-\hat{x}\right\|_{H_{n}^{1}} & =O(\varepsilon), \\
\left\|\hat{u}_{\varepsilon}-\hat{u}\right\|_{L_{m}^{2}} & =O(\varepsilon),
\end{aligned}
$$

as $\varepsilon \downarrow 0$. The analysis of these estimates is central to our development.

For each $f \in L_{n}^{2}$, let $(\hat{x}(f), \hat{u}(f)) \in H_{0 n}^{1} \times L_{m}^{2}$ be the solution of $(0.1)$ and (0.2) corresponding to this $f$. This induces a mapping $\mathcal{L}: L_{n}^{2} \rightarrow H_{0 n}^{1} \times L_{m}^{2}$ defined by

$$
\mathcal{L}(f) \equiv(\hat{x}(f), \hat{u}(f)) \text {. }
$$

The following lemma states that the optimal control problem $(0.1)$ and $(0.2)$ is well-posed with respect to the inhomogeneous data $f$.

Lemma 1. Let (0.3) and (0.6) hold. Then the mapping $\mathcal{L}$ defined by (1.4) is a bounded linear transformation from $L_{n}^{2}$ into $H_{0 n}^{1} \times L_{m}^{2}$.

Proof. This can be easily verified from the variational equation $(0.4)$ satisfied by $\hat{u}$, using the primal theory.

Let $f_{\varepsilon} \equiv \dot{\hat{x}}_{\varepsilon}-A \hat{x}_{\varepsilon}-B \hat{u}_{\varepsilon}$. It is obvious that $\left(\hat{x}_{\varepsilon}, \hat{u}_{\varepsilon}\right)$ is the unique solution of

$\operatorname{Min} J(x, u)$

Subject to

$$
\left\{\begin{array}{l}
\dot{x}(t)=A x(t)+B u(t)+f_{\varepsilon}(t), \quad t \in[0, T], \\
x(0)=0
\end{array}\right.
$$

This implies that

$$
\mathcal{L}\left(f_{\varepsilon}\right)=\left(\hat{x}_{\varepsilon}, \hat{u}_{\varepsilon}\right)
$$

Therefore, by Lemma 1 ,

$$
\left\|\hat{x}-\hat{x}_{\varepsilon}\right\|_{H_{0 n}^{1}}+\left\|\hat{u}-\hat{u}_{\varepsilon}\right\|_{L_{m}^{2}}=\left\|\mathscr{L}(f)-\mathcal{L}\left(f_{\varepsilon}\right)\right\|_{H_{0 n}^{1} \times L_{m}^{2}} \leqslant\|\mathscr{L}\|\left\|f-f_{\varepsilon}\right\|_{L_{n}^{2}} .
$$

Since $\left(\hat{x}_{\varepsilon}, \hat{u}_{\varepsilon}\right)$ minimizes $J_{\varepsilon}$, we have

$$
\begin{aligned}
J\left(\hat{x}_{\varepsilon}, \hat{u}_{\varepsilon}\right) & \leqslant J_{\varepsilon}\left(\hat{x}_{\varepsilon}, \hat{u}_{\varepsilon}, f\right)=J\left(\hat{x}_{\varepsilon}, \hat{u}_{\varepsilon}\right)+\frac{1}{\varepsilon}\left\|\dot{\hat{x}}_{\varepsilon}-A \hat{x}_{\varepsilon}-B \hat{u}_{\varepsilon}-f\right\|_{L_{n}^{2}}^{2} \\
& \leqslant J_{\varepsilon}(\hat{x}, \hat{u}, f)=J(\hat{x}, \hat{u}) .
\end{aligned}
$$


Thus

$$
\text { (1.8) } \begin{aligned}
\frac{1}{\varepsilon}\left\|\dot{\hat{x}}_{\varepsilon}-A \hat{x}_{\varepsilon}-B \hat{u}_{\varepsilon}-f\right\|_{L_{n}^{2}}^{2} & =\frac{1}{\varepsilon}\left\|f_{\varepsilon}-f\right\|_{L_{n}^{2}}^{2} \leqslant J(\hat{x}, \hat{u})-J\left(\hat{x}_{\varepsilon}, \hat{u}_{\varepsilon}\right) \\
& =2 \cdot a\left(\left[\begin{array}{c}
\hat{x}_{\varepsilon} \\
\hat{u}_{\varepsilon}
\end{array}\right],\left[\begin{array}{c}
\hat{x}-\hat{x}_{\varepsilon} \\
\hat{u}-\hat{u}_{\varepsilon}
\end{array}\right]\right)+J\left(\hat{x}-\hat{x}_{\varepsilon}, \hat{u}-\hat{u}_{\varepsilon}\right) .
\end{aligned}
$$

Therefore

$$
\begin{aligned}
\frac{1}{\varepsilon}\left\|f_{\varepsilon}-f\right\|^{2} \leqslant & K\left(\left\|\left(\hat{x}_{\varepsilon}, \hat{u}_{\varepsilon}\right)\right\|_{H_{0 n}^{1} \times L_{m}^{2}}\left\|\left(\hat{x}-\hat{x}_{\varepsilon}, \hat{u}-\hat{u}_{\varepsilon}\right)\right\|_{H_{0 n}^{1} \times L_{m}^{2}}\right) \\
& +K\left(\left\|\left(\hat{x}-\hat{x}_{\varepsilon}, \hat{u}-\hat{u}_{\varepsilon}\right)\right\|_{H_{0 n}^{1} \times L_{m}^{2}}^{2}\right),
\end{aligned}
$$

where $K$ is a positive constant satisfying

$$
\begin{gathered}
2\left|a\left(\left[\begin{array}{l}
y_{1} \\
v_{1}
\end{array}\right],\left[\begin{array}{l}
y_{2} \\
v_{2}
\end{array}\right]\right)\right| \leqslant K\left\|\left(y_{1}, v_{1}\right)\right\|_{H_{0 n}^{1} \times L_{m}^{2}}\left\|\left(y_{2}, v_{2}\right)\right\|_{H_{0_{n}}^{1} \times L_{m}^{2}}, \\
J(y, v) \leqslant K\|(y, v)\|_{H_{0 n}^{1} \times L_{m}^{2} .}^{2}
\end{gathered}
$$

By (1.6) we obtain

$$
\begin{aligned}
\frac{1}{\varepsilon}\left\|f_{\varepsilon}-f\right\|_{L_{n}^{2}}^{2} & \leqslant K\left(\left\|\left(\hat{x}_{\varepsilon}, \hat{u}_{\varepsilon}\right)\right\|+\left\|\left(\hat{x}-\hat{x}_{\varepsilon}, \hat{u}-\hat{u}_{\varepsilon}\right)\right\|\right)\left\|\left(\hat{x}-\hat{x}_{\varepsilon}, \hat{u}-\hat{u}_{\varepsilon}\right)\right\| \\
& \leqslant K\left(\left\|\left(\hat{x}_{\varepsilon}, \hat{u}_{\varepsilon}\right)\right\|+\left\|\left(\hat{x}-\hat{x}_{\varepsilon}, \hat{u}-\hat{u}_{\varepsilon}\right)\right\|\right)\|\varrho\|\left\|f_{\varepsilon}-f\right\|_{L_{n}^{2} .}
\end{aligned}
$$

So

$$
\left\|f_{\varepsilon}-f\right\|_{L_{n}^{2}} \leqslant K\left(\left\|\left(\hat{x}_{\varepsilon}, \hat{u}_{\varepsilon}\right)\right\|+\left\|\left(\hat{x}-\hat{x}_{\varepsilon}, \hat{u}-\hat{u}_{\varepsilon}\right)\right\|\right)\|\varrho\| \cdot \varepsilon .
$$

By (1.7), we have

$$
\begin{aligned}
\left\|\left(\hat{x}_{\varepsilon}, \hat{u}_{\varepsilon}\right)\right\| & \leqslant\left[\frac{1}{\nu} J\left(\hat{x}_{\varepsilon}, \hat{u}_{\varepsilon}\right)\right]^{1 / 2} \leqslant\left[\frac{1}{\nu} J(\hat{x}, \hat{u})\right]^{1 / 2} \\
& \leqslant\left[\frac{1}{\nu} K\|(\hat{x}, \hat{u})\|^{2}\right]^{1 / 2}=\nu^{-1 / 2} K^{1 / 2}\|(\hat{x}, \hat{u})\|,
\end{aligned}
$$

where $\nu$ is the positive constant in (0.3). So, using (1.6), (1.9), and (1.10) we get

$$
\begin{aligned}
& \left\|\hat{x}_{\varepsilon}-\hat{x}\right\|_{H_{0 n}^{1}}+\left\|\hat{u}_{\varepsilon}-\hat{u}\right\|_{L_{m}^{2}} \leqslant\|\mathcal{L}\|\left\|f_{\varepsilon}-f\right\|_{L_{n}^{2}}
\end{aligned}
$$

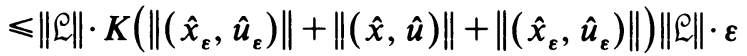

$$
\begin{aligned}
& \leqslant\|\varrho\|^{2} \cdot K \cdot \varepsilon\left[\left(1+2 \nu^{-1 / 2} K^{1 / 2}\right)\|(\hat{x}, \hat{u})\|\right] \\
& \equiv \bar{K} \cdot\|(\hat{x}, \hat{u})\|\|\mathcal{L}\|^{2} \cdot \varepsilon, \quad \bar{K} \equiv K\left(1+2 \nu^{-1 / 2} K^{1 / 2}\right) \text {. }
\end{aligned}
$$

We summarize the above in

THEOREM 2. Let $\left(\hat{x}_{\varepsilon}, \hat{u}_{\varepsilon}\right)$ solve $(1.1)$ and $(\hat{x}, \hat{u})$ solve $(0.1),(0.2)$. If $(0.3)$ and $(0.6)$ hold, then

$$
\left\|\hat{x}_{\varepsilon}-\hat{x}\right\|_{H_{0 n}^{1}}+\left\|\hat{u}_{\varepsilon}-\hat{u}\right\|_{L_{m}^{2}} \leqslant \bar{K} \cdot\|(\hat{x}, \hat{u})\|\|\varrho\|^{2} \cdot \varepsilon
$$

for all $\varepsilon>0$, where $\bar{K}>0$ is independent of $\hat{x}, \hat{u}$ and $\varepsilon$.

For any $f \in L_{n}^{2}$, define $\mathcal{L}_{\varepsilon}: L_{n}^{2} \rightarrow H_{0 n}^{1} \times L_{m}^{2}$ by $\mathcal{L}_{\varepsilon}(f) \equiv\left(\hat{x}_{\varepsilon}, \hat{u}_{\varepsilon}\right)$, where $\left(\hat{x}_{\varepsilon}, \hat{u}_{\varepsilon}\right)$ is the unique solution of (1.1) corresponding to this $f$. From (1.5) we have $\mathcal{L}_{\varepsilon}(f)=\mathcal{L}\left(f_{\varepsilon}\right)$. 
Lemma 3. Let (0.3) and (0.6) hold. Then the mapping $\mathcal{L}_{\varepsilon}$ is a bounded linear transformation from $L_{n}^{2}$ into $H_{0 n}^{1} \times L_{m}^{2}$. Furthermore,

$$
\varlimsup_{\varepsilon \downarrow 0}\left\{\left\|\mathcal{L}_{\varepsilon}\right\|\right\}<\infty \text {. }
$$

Proof. The fact that $\mathcal{L}_{\varepsilon}$ is bounded linear can be verified from the variational equation for (1.1), see (1.13) below. By Theorem 2, we have

$$
\varrho_{\varepsilon} f \rightarrow \varrho f \text { as } \varepsilon \downarrow 0, \quad \forall f \in L_{n}^{2} .
$$

Applying the uniform boundedness principle gives (1.11).

Remark 4. The penalized problem (1.1) is equivalent to the variational equation

$$
a_{\varepsilon}\left(\left[\begin{array}{l}
\hat{x}_{\varepsilon} \\
\hat{u}_{\varepsilon}
\end{array}\right],\left[\begin{array}{l}
y \\
v
\end{array}\right]\right)=\theta_{\varepsilon}\left(\left[\begin{array}{l}
y \\
v
\end{array}\right]\right), \quad \forall\left[\begin{array}{l}
y \\
v
\end{array}\right] \in H_{0 n}^{1} \times L_{m}^{2},
$$

where

$$
a_{\varepsilon}\left(\left[\begin{array}{l}
x_{1} \\
u_{1}
\end{array}\right],\left[\begin{array}{l}
x_{2} \\
u_{2}
\end{array}\right]\right) \equiv a\left(\left[\begin{array}{l}
x_{1} \\
u_{1}
\end{array}\right],\left[\begin{array}{l}
x_{2} \\
u_{2}
\end{array}\right]\right)+\frac{1}{\varepsilon}\left\langle\dot{x}_{1}-A x_{1}-B u_{1}, \dot{x}_{2}-B x_{2}-B u_{2}\right\rangle_{L_{n}^{2}}
$$

and

$$
\theta_{\varepsilon}\left(\left[\begin{array}{l}
x \\
u
\end{array}\right]\right) \equiv \frac{1}{\varepsilon}\langle f, \dot{x}-A x-B u\rangle_{L_{n}^{2}}
$$

From [10], we know that

$$
s-\lim _{\varepsilon \downarrow 0} \frac{1}{\varepsilon}\left(\dot{\hat{x}}_{\varepsilon}-A \hat{x}_{\varepsilon}-B \hat{u}_{\varepsilon}-f\right)=\hat{p} \quad \text { in } L_{n}^{2}
$$

for some $\hat{p} \in L_{n}^{2}$, which is the Lagrange multiplier. In the limit (1.13) becomes

$$
a\left(\left[\begin{array}{l}
\hat{x} \\
\hat{u}
\end{array}\right],\left[\begin{array}{l}
y \\
v
\end{array}\right]\right)+\langle\hat{p}, \dot{y}-A y-B v\rangle_{L_{n}^{2}}=0 \quad \forall(y, v) \in H_{0 n}^{1} \times L_{m}^{2},
$$

the variational equation for $(\hat{x}, \hat{u})$.

We now approximate the penalized problem (1.1) by finite elements. Let $\left\{S_{h}^{1} \times\right.$ $\left.S_{h}^{2} \mid 0 \leqslant h \leqslant h_{0}\right\}$ be a one-parameter family of products of finite-dimensional subspaces $S_{h}^{1}$ and $S_{h}^{2}$ satisfying

$$
\left\{\begin{array}{l}
S_{h}^{1} \times S_{h}^{2} \subseteq H_{0 n}^{1} \times L_{m}^{2}, \\
\lim _{h \downarrow 0} \inf _{y_{h} \in S_{h}^{1}}\left\|y-y_{h}\right\|_{H_{0 n}^{1}}=0 \quad \text { for any } y \in H_{0 n}^{1}, \\
\lim _{h \downarrow 0} \inf _{v_{h} \in S_{h}^{2}}\left\|v-v_{h}\right\|_{L_{m}^{2}}=0 \quad \text { for any } v \in L_{m}^{2} .
\end{array}\right.
$$

The approximation is to solve (1.1) over $S_{h}^{1} \times S_{h}^{2}$. For each $\varepsilon>0, h>0$ let $\left(\hat{x}_{h}^{\varepsilon}, \hat{u}_{h}^{\varepsilon}\right)$ be the unique solution of

$$
\operatorname{Min}_{(x, u) \in S_{h}^{1} \times S_{h}^{2}} J_{\varepsilon}(x, u, f) .
$$

This is equivalent to the variational problem

$$
a_{\varepsilon}\left(\left[\begin{array}{c}
\hat{x}_{h}^{\varepsilon} \\
\hat{u}_{h}^{\varepsilon}
\end{array}\right],\left[\begin{array}{l}
v_{1} \\
v_{2}
\end{array}\right]\right)=\theta_{\varepsilon}\left(\left[\begin{array}{l}
v_{1} \\
v_{2}
\end{array}\right]\right) \quad \forall\left(v_{1}, v_{2}\right) \in S_{h}^{1} \times S_{h}^{2}
$$


If $\left\{\psi_{i}\right\}_{i=1}^{K_{1}},\left\{\varphi_{i}\right\}_{i=1}^{K_{2}}$ are bases for $S_{h}^{1}, S_{h}^{2}$, respectively, (1.17) is a matrix equation $M_{\varepsilon, h} \bar{q}_{\varepsilon, h}=\bar{\theta}_{\varepsilon, h}$, where

$$
\left[M_{\varepsilon, h}\right]_{i j}=a_{\varepsilon}\left(\Phi_{j}, \Phi_{i}\right), \quad\left(\bar{\theta}_{\varepsilon, h}\right)_{j}=\theta_{\varepsilon}\left(\Phi_{j}\right),
$$

and $\left\{\Phi_{i}\right\}_{i=1}^{K_{1}+K_{2}}$ is the basis for $S_{h}^{1} \times S_{h}^{2}$ induced by $\left\{\psi_{i}\right\}$ and $\left\{\varphi_{i}\right\}$. More specifically,

$$
M_{\varepsilon, h}=\left[\begin{array}{l:l}
\frac{1}{\varepsilon} \int_{0}^{T}\left\langle\dot{\psi}_{j}-A \psi_{j}, \dot{\psi}_{i}-A \psi_{i}\right\rangle_{\mathbf{R}^{n}} d t & -\frac{1}{\varepsilon} \int_{0}^{T}\left\langle B \varphi_{j}, \dot{\psi}_{i}-A \psi_{i}\right\rangle_{\mathbf{R}^{n}} d t \\
+\int_{0}^{T}\left[\left\langle\psi_{j}, N_{2} \psi_{i}\right\rangle_{\mathbf{R}^{n}}+\left\langle\dot{\psi}_{j}, N_{1} \dot{\psi}_{i}\right\rangle_{\mathbf{R}^{n}}\right] d t & \\
\hdashline-\frac{1}{\varepsilon} \int_{0}^{T}\left\langle\dot{\psi}_{j}-A \psi_{j}, B \varphi_{i}\right\rangle_{\mathbf{R}^{n}} d t & \frac{1}{\varepsilon} \int_{0}^{T}\left\langle B \varphi_{j}, B \varphi_{i}\right\rangle_{\mathbf{R}^{n}} d t
\end{array}\right]
$$

and

$$
\bar{\theta}_{\varepsilon, h}=\left[\begin{array}{c}
\frac{1}{\varepsilon} \int_{0}^{T}\left\langle f, \dot{\psi}_{i}-A \psi_{i}\right\rangle_{\mathbf{R}^{n}} d t \\
--\frac{1}{\varepsilon} \int_{0}^{T}\left\langle f, B \phi_{i}\right\rangle_{\mathbf{R}^{n}} d t
\end{array}\right]
$$

Examples in Section 4 show that this matrix is of a block banded structure for certain choices of approximating spaces.

The analysis of the errors in this approximation is quite subtle. We begin by introducing certain subspaces of $L_{n}^{2}$ associated with $S_{h}^{1}$ and $S_{h}^{2}$. We define

$$
\begin{aligned}
V_{h}^{1} & \equiv\left\{\dot{y}_{h}-A y_{h} \mid y_{h} \in S_{h}^{1}\right\}, \\
V_{h}^{2} & \equiv\left\{B v_{h} \mid v_{h} \in S_{h}^{2}\right\} .
\end{aligned}
$$

We denote by $V_{h}^{1}+V_{h}^{2}$ the closed linear span of $V_{h}^{1} \cup V_{h}^{2}$. Then $V_{h}^{1}+V_{h}^{2}$ becomes a finite-dimensional subspace of $L_{n}^{2}$. It is easy to verify that

$$
\lim _{h \downarrow 0} \inf _{w_{h} \in V_{h}^{1}+V_{h}^{2}}\left\|w-w_{h}\right\|_{L_{n}^{2}}=0
$$

is satisfied, for all $w \in L_{n}^{2}$, provided (1.15) holds.

For a given Hilbert space $H$ with some closed subspace $H_{1}$, we let $\mathbf{P}_{H_{1}}$ denote the orthogonal projection of $H$ onto $H_{1}$. The error analysis hinges on the behavior of the operators $\mathcal{L}_{h}^{\varepsilon}: L_{n}^{2} \rightarrow H_{0 n}^{1} \times L_{m}^{2}$ defined by

$$
\mathcal{L}_{h}^{\varepsilon}(f) \equiv\left(\hat{x}_{h}^{\varepsilon}, \hat{u}_{h}^{\varepsilon}\right)
$$

where $\left(\hat{x}_{h}^{\varepsilon}, \hat{u}_{h}^{\varepsilon}\right)$ solves (1.16).

LEMMA 5. Let (0.3), (0.6), and (1.15) hold. Then the mapping $\mathcal{L}_{h}^{\varepsilon}$ defined above is a bounded linear transformation from $L^{2}$ into $H_{0 n}^{1} \times L_{m}^{2}$ with $\operatorname{Range}\left(\mathcal{L}_{h}^{\varepsilon}\right) \subseteq S_{h}^{1} \times S_{h}^{2}$. $\left(\hat{x}_{h}^{\varepsilon}, \hat{u}_{h}^{\varepsilon}\right)$, the image of $f$ under $\mathcal{L}_{h}^{\varepsilon}$, is characterized by the variational equation

$$
a_{\varepsilon}\left(\left[\begin{array}{c}
\hat{x}_{h}^{\varepsilon} \\
\hat{u}_{h}^{\varepsilon}
\end{array}\right],\left[\begin{array}{c}
y_{h} \\
v_{h}
\end{array}\right]\right)=\theta_{\varepsilon}\left(f_{h}\right)
$$

for all $\left(y_{h}, v_{h}\right) \in S_{h}^{1} \times S_{h}^{2}$, where $f_{h} \equiv \mathbf{P}_{V_{h}^{1}+V_{h}^{2}} f$. 
Proof. Since $\left\langle f, \dot{y}_{h}-A y_{h}-B v_{h}\right\rangle=\left\langle f_{h}, \dot{y}_{h}-A y_{h}-B v_{h}\right\rangle$, we replace $f$ by $f_{h}$ in (1.17) and get (1.21).

An immediate consequence of the above lemma is

$$
\mathcal{L}_{h}^{\varepsilon}(f)=\mathcal{E}_{h}^{\varepsilon}\left(f_{h}\right) \text {. }
$$

In solving (1.16), unless $f \in V_{h}^{1}+V_{h}^{2}$, it is in general true that

$$
J_{\varepsilon}\left(\hat{x}_{h}^{\varepsilon}, \hat{u}_{h}^{\varepsilon}, f\right) \rightarrow \infty, \quad \text { as } \varepsilon \downarrow 0,
$$

because no $\left(y_{h}, v_{h}\right)$ in $S_{h}^{1} \times S_{h}^{2}$ can satisfy the constraint $\dot{y}_{h}-A y_{h}-B v_{h}-f=0$. This makes one wonder whether the solution $\left(\hat{x}_{h}^{\varepsilon}, \hat{u}_{h}^{\varepsilon}\right)$ in Lemma 5 will converge as $\varepsilon \downarrow 0$. However, solving (1.16) is equivalent to solving

$$
\operatorname{Min}_{(x, u) \in S_{h}^{1} \times S_{h}^{2}} J_{\varepsilon}\left(x, u, f_{h}\right) .
$$

Because solutions $\left(\hat{x}_{h}^{\varepsilon}, \hat{u}_{h}^{e}\right)$ of $(1.24)$ do converge as $\varepsilon \downarrow 0$, we conclude that

$$
s-\lim _{\varepsilon \downarrow 0}\left(\hat{x}_{h}^{\varepsilon}, \hat{u}_{h}^{\varepsilon}\right)=\left(\hat{x}_{h}, \hat{u}_{h}\right) \text { in } H_{0 n}^{1} \times L_{m}^{2}
$$

for some unique $\left(\hat{x}_{h}, \hat{u}_{h}\right)$. This defines a mapping $\varrho_{h}$ by

$$
\mathcal{L}_{h}: L_{n}^{2} \rightarrow H_{0 n}^{1} \times L_{m}^{2}, \quad \mathcal{L}_{h}(f) \equiv\left(\hat{x}_{h}, \hat{u}_{h}\right) .
$$

Then Range $\left(\mathcal{L}_{n}\right) \subset S_{h}^{1} \times S_{h}^{2}$. Arguing in the same manner as in the proof of Theorem 2, we have

COROllaRy 6. Let (0.3), (0.6), and (1.15) hold. Then, for each $h>0$, the mapping $\mathcal{L}_{h}$ defined above is a bounded linear transformation from $L_{n}^{2}$ into $H_{0 n}^{1} \times L_{m}^{2}$ with Range $\left(\mathcal{L}_{h}\right) \subseteq S_{h}^{1} \times S_{h}^{2} .\left(\hat{x}_{h}, \hat{u}_{h}\right)$, the image of $f$ under $\mathcal{L}_{h}$, is the unique solution of

$$
\left\{\begin{array}{l}
\operatorname{Min} J(x, u) \\
\text { subject to } \\
(x, u) \in S_{h}^{1} \times S_{h}^{2} \\
\dot{x}=A x+B u+f_{h}, \quad f_{h}=\mathbf{P}_{V_{h}^{1}+V_{h}^{2} f} \\
x(0)=0 .
\end{array}\right.
$$

Furthermore, for any $f \in L_{n}^{2}$, we have

$$
\left\|\hat{x}_{h}^{\varepsilon}-\hat{x}_{h}\right\|_{H_{0 n}^{1}}+\left\|\hat{u}_{h}^{\varepsilon}-\hat{u}_{h}\right\|_{L_{m}^{2}} \leqslant \bar{K} \cdot\left\|\left(\hat{x}_{h}, \hat{u}_{h}\right)\right\|_{H_{0 n}^{1} \times L_{m}^{2}} \cdot\left\|\mathcal{L}_{n}\right\|^{2} \cdot \varepsilon
$$

for all $\varepsilon>0$.

It is obvious that the properties

$$
\varrho_{h}(f)=e_{h}\left(f_{h}\right), \quad \dot{\hat{x}}_{h}-A \hat{x}_{h}-B \hat{u}_{h}=f_{h}=\mathbf{P}_{V_{h}^{1}+V_{h}^{2}} f
$$

are satisfied for all $f \in L_{n}^{2}$. Also, the analogue of (1.14) for problem (1.16) is

$$
a\left(\left[\begin{array}{l}
\hat{x}_{h} \\
\hat{u}_{h}
\end{array}\right],\left[\begin{array}{l}
y_{h} \\
v_{h}
\end{array}\right]\right)+\left\langle\hat{p}_{h}, \dot{y}_{h}-A y_{h}-B v_{h}\right\rangle_{L_{n}^{2}}=0 \quad \forall\left(y_{h}, v_{h}\right) \in S_{h}^{1} \times S_{h}^{2} .
$$

2. An Abstract Approximation Problem. In order to obtain optimal error estimates for solutions of (1.16), it is necessary to consider the following abstract approximation problem: "Let $\left\{G_{i}^{1}\right\},\left\{G_{i}^{2}\right\}$ be two families of increasing finite-dimensional subspaces of a Hilbert space $H$ such that

$$
\lim _{i \rightarrow \infty} \inf _{g_{i} \in G_{i}^{1}}\left\|x-g_{i}\right\|_{H}=0 \quad \forall x \in H .
$$




$$
\left\{\begin{array}{l}
G_{i}^{1} \subseteq G_{j}^{1}, G_{i}^{2} \subseteq G_{j}^{2} \text { for } i<j, \\
G_{i_{k_{1}}}^{1} \subsetneq G_{i_{k_{2}}}^{1}, G_{i_{k_{1}}}^{2} \subsetneq G_{i_{k_{2}}}^{2} \text { if } k_{1}<k_{2}, \text { for some increasing sequence }\left\{i_{k}\right\} \subseteq \mathbf{N} .
\end{array}\right.
$$

Then, for any $f \in H$, does there always exist a sequence

$$
\left\{\left(g_{i}^{1}, g_{i}^{2}\right) \mid g_{i}^{1} \in G_{i}^{1}, g_{i}^{2} \in G_{i}^{2}\right\}_{i=1}^{\infty}
$$

satisfying

$$
\mathbf{P}_{G_{i}^{1}+G_{i}^{2}} f=g_{i}^{1}+g_{i}^{2}
$$

such that

$$
\varlimsup_{i \rightarrow \infty}\left[\left\|g_{i}^{1}\right\|+\left\|g_{i}^{2}\right\|\right]<\infty ? ” .
$$

Since this problem has quite independent interest, we study it separately in this section. In Section 3, we will apply these results using $G_{i}^{1}=V_{h_{i}}^{1}$ and $G_{i}^{2}=V_{h_{i}}^{2}$, the spaces defined in (1.18) and (1.19).

We let

$$
\tilde{G}_{i}^{2} \equiv G_{i}^{2} \ominus\left(G_{i}^{1} \cap G_{i}^{2}\right)
$$

be the orthogonal complement of $G_{i}^{1} \cap G_{i}^{2}$ in $G_{i}^{2}$ and form the orthogonal decomposition

$$
G_{i}^{2}=\left(G_{i}^{1} \cap G_{i}^{2}\right) \oplus \tilde{G}_{i}^{2}
$$

Then

$$
G_{i}^{1}+G_{i}^{2}=G_{i}^{1}+\tilde{G}_{i}^{2}
$$

holds, where the right-hand side becomes a (in general, nonorthogonal) direct sum.

Definition 7. For any two closed subspaces $H_{1}$ and $H_{2}$ of a Hilbert space $H$, we define [8]

$$
\cos \left(H_{1}, H_{2}\right) \equiv \begin{cases}\sup _{\left\|f_{1}\right\|=\left\|f_{2}\right\|=1}\left|\left\langle f_{1}, f_{2}\right\rangle\right| & \text { if } H_{1} \neq\{0\}, H_{2} \neq\{0\}, \\ 0 & \text { if } H_{1}=\{0\} \text { or } H_{2}=\{0\} .\end{cases}
$$

THEOREM 8. Let $\left\{G_{i}^{1}\right\}_{i}$ and $\left\{G_{i}^{2}\right\}_{i}$ be two sequences of finite-dimensional subspaces in $H$ satisfying (2.1) and (2.2) For any $f \in H$, there exists a sequence $\left\{\left(g_{i}^{1}, g_{i}^{2}\right) \in G_{i}^{1} \times\right.$ $G_{i}^{2} \mid(2.3)$ holds s satisfying (2.4) if and only if there exists some $\mu \geqslant 0$ such that

$$
\cos \left(G_{i}^{1}, \tilde{G}_{i}^{2}\right) \leqslant \mu<1 \quad \forall i \in \mathbf{Z}^{+} .
$$

Proof. (only if) Assume the contrary that (2.8) does not hold. Then there exists a sequence $\left\{i_{j}\right\}$ such that

$$
\cos \left(G_{i_{j}}^{1}, \tilde{G}_{i_{j}}^{2}\right) \equiv \mu_{i_{j}} \rightarrow 1 \quad \text { as } j \rightarrow \infty .
$$

This is easily seen to be equivalent to

$$
\inf _{\substack{\|x\|=1 \\ x \in \tilde{G}_{i j}^{2}}}\left\|x-\mathbf{P}_{G_{i_{j}}^{\prime}} x\right\| \equiv \alpha_{i_{j}} \rightarrow 0 \quad \text { as } j \rightarrow \infty .
$$


Here we assume that $\tilde{G}_{i j}^{2}$ is nontrivial ( $Z\{0\}$ ), thus $\alpha_{i_{j}}>0$. Because $\tilde{G}_{i_{j}}^{2}$ is finitedimensional, (2.10) attains its minimum at some $x_{i_{j}} \in \tilde{G}_{i_{j}}^{2}$ :

$$
\left\|x_{i_{j}}-\mathbf{P}_{G_{i_{j}}^{1}} x_{i_{j}}\right\|=\inf _{\substack{\|x\|=1 \\ x \in \tilde{G}_{i_{j}}^{2}}}\left\|x-\mathbf{P}_{G_{i_{j}}^{1}} x\right\|=\alpha_{i_{j}}, \quad\left\|x_{i_{j}}\right\|=1 .
$$

$$
\lim _{j \rightarrow \infty} \alpha_{i_{j}}=0 .
$$

For any $x \in G_{i}^{1}+G_{i}^{2}$, by (2.7), we have a unique representation

$$
x=g_{i}^{1}(x)+g_{i}^{2}(x), \quad g_{i}^{1}(x) \in G_{i}^{1}, g_{i}^{2}(x) \in \tilde{G}_{i}^{2} .
$$

We define

$$
P_{i}: G_{i}^{1}+G_{i}^{2} \rightarrow G_{i}^{1}, \quad P_{i} x \equiv g_{i}^{(1)}(x),
$$

and let $\left\|P_{i}\right\|_{\mathcal{L}\left(G_{i}^{1}+G_{i}^{2}, G_{i}^{l}\right)} \equiv \gamma_{i}, i \in \mathbf{Z}^{+}$. Note here that $\gamma_{i} \geqslant 1$.

Now we choose a subsequence $\left\{i_{k}\right\}_{k \in \mathbf{Z}^{+}}$of positive integers and a sequence $\left\{\beta_{k}\right\}_{k \in \mathbf{Z}^{+}}$of increasing positive real numbers with the following properties:

$$
\left\{\begin{array}{l}
\beta_{k} \equiv\left(\alpha_{i_{k}} 2^{k}\right)^{-1 / 3} \alpha_{1}^{1 / 3}, \quad k \in \mathbf{Z}^{+}, \alpha_{i_{1}} \equiv \alpha_{1}, \\
\beta_{k} \geqslant \gamma_{i_{l}} \quad(1 \leqslant l<k), k \in \mathbf{Z}^{+}, \\
\lim _{k \rightarrow \infty}\left[\beta_{k}-\left(1+\gamma_{i_{k}}\right) \sum_{j=1}^{k-1} \beta_{j}\right]=+\infty .
\end{array}\right.
$$

The constructability of such sequences $\left\{i_{k}\right\}$ and $\left\{\beta_{k}\right\}$ is guaranteed by (2.12).

Let

$$
f_{0} \equiv \sum_{k=1}^{\infty} \beta_{k}\left(x_{i_{k}}-\mathbf{P}_{G_{i_{k}}^{1}} x_{i_{k}}\right),
$$

where $x_{i_{k}} \in \tilde{G}_{i_{k}}^{2}$ is defined through (2.11). Using (2.12)-(2.14), we easily verify that $f_{0} \in H$.

Let

$$
\xi_{l} \equiv \sum_{j=1}^{l} \beta_{j}\left(x_{i_{j}}-\mathbf{P}_{G_{i_{j}}^{\prime}} x_{i_{j}}\right), \quad \eta_{l} \equiv f_{0}-\xi_{l} .
$$

Since $\xi_{l} \in G_{i_{l}}^{1}+G_{i_{l}}^{2}$, we have

$$
\begin{aligned}
\mathbf{P}_{G_{i}^{1}}+G_{i_{l}}^{2} f_{0}= & \mathbf{P}_{G_{i_{l}}^{1}+G_{i_{l}}^{2}}\left[\xi_{l}+\eta_{l}\right]=\xi_{l}+\mathbf{P}_{G_{i_{l}}^{1}+G_{i_{l}}^{2}}\left[\eta_{l}\right] \\
= & \left(I-P_{i_{l}}\right)\left[\beta_{l} x_{i_{l}}+\sum_{j=1}^{l-1} \beta_{j} x_{i_{j}}+\mathbf{P}_{G_{i_{l}}^{1}+G_{i_{l}}^{2}} \eta_{l}\right] \\
& +\left[P_{i_{l}}\left(\sum_{j=1}^{l} \beta_{j} x_{i_{j}}+\mathbf{P}_{G_{i_{l}}^{1}+G_{i_{l}}^{2}} \eta_{l}\right)-\sum_{j=1}^{l} \beta_{j} \mathbf{P}_{G_{i_{j}}^{1}} x_{i_{j}}\right] .
\end{aligned}
$$

In the above, the first term belongs to $\tilde{G}_{i_{i}}^{2}$ and the second belongs to $G_{i}^{1}$. Thus we have the unique representation

$$
\mathbf{P}_{G_{i}^{1}}+G_{i,}^{2} f_{0}=(2.17) \equiv g_{i,}^{2}\left(f_{0}\right)+g_{i}^{1}\left(f_{0}\right) .
$$


We wish to show that $\lim _{l \rightarrow \infty}\left\|g_{i}^{2}\left(f_{0}\right)\right\|=\infty$. From (2.16), (2.13), and (2.14),

$$
\begin{aligned}
\left\|\eta_{l}\right\| & \leqslant \sum_{j=l+1}^{\infty} \beta_{j}\left\|x_{i_{j}}-\mathbf{P}_{G_{i j}} x_{i_{j}}\right\|=\sum_{j=l+1}^{\infty} \beta_{j} \alpha_{i_{j}}=\sum_{j=l+1}^{\infty} \frac{\alpha_{1}}{\beta_{j}^{2} 2^{j}} \\
& \leqslant \frac{\alpha_{1}}{\beta_{l+1}^{2}} \leqslant \frac{\alpha_{1}}{\beta_{l+1}} \frac{1}{\gamma_{i_{l}}}=\frac{\alpha_{1}}{\beta_{l+1}}\left\|P_{i_{l}}\right\|^{-1}
\end{aligned}
$$

Because $x_{i_{l}} \in \tilde{G}_{i_{l}}^{2},\left(I-P_{i_{l}}\right) x_{i_{l}}=x_{i_{l}}$, and because $\mathbf{P}_{G_{i l}^{1}+G_{i_{l}}^{2}}$ is an orthogonal projection, (2.13), (2.17)-(2.19) give

$$
\begin{aligned}
\left\|g_{i_{l}}^{2}\left(f_{0}\right)\right\| & \geqslant\left\|\beta_{l} x_{i_{l}}\right\|-\left\|\left(I-P_{i_{l}}\right) \sum_{j=1}^{l-1} \beta_{j} x_{i_{j}}\right\|-\left\|\left(I-P_{i_{l}}\right) \mathbf{P}_{G_{i_{l}}+G_{i_{l}}^{2}} \eta_{l}\right\| \\
& \geqslant\left[\beta_{l}-\left(1+\left\|P_{i_{l}}\right\|\right) \sum_{j=1}^{l-1} \beta_{j}\right]-\left(1+\left\|P_{i_{l}}\right\|\right) \frac{\alpha_{1}}{\beta_{l+1}}\left\|P_{i_{l}}\right\|^{-1} .
\end{aligned}
$$

The bracketed term in (2.20) tends to $+\infty$ as $l \rightarrow \infty$ by (2.14), and $\left\|P_{i_{l}}\right\| \geqslant 1$ gives

$$
\left(1+\left\|P_{i},\right\|\right) \frac{\alpha_{1}}{\beta_{l+1}}\left\|P_{i}\right\|^{-1} \rightarrow 0 \quad \text { as } l \rightarrow \infty \text {. }
$$

Hence

$$
\lim _{l \rightarrow \infty}\left\|g_{i_{l}}^{2}\left(f_{0}\right)\right\|=\infty
$$

For this $f_{0}$, if there still exist sequences $\left\{\bar{g}_{i}^{1} \in G_{i}^{1}\right\},\left\{\bar{g}_{i}^{2} \in G_{i}^{2}\right\}$ satisfying (2.3) and (2.4), then

$$
\mathbf{P}_{G_{i}^{1}}+G_{i_{l}}^{2} f_{0}=(2.17)=g_{i_{l}}^{1}\left(f_{0}\right)+g_{i_{l}}^{2}\left(f_{0}\right)=(2.3)=\bar{g}_{i_{l}}^{1}+\bar{g}_{i_{l}}^{2} \text {. }
$$

Therefore

$$
g_{i_{l}}^{1}\left(f_{0}\right)-\bar{g}_{i_{l}}^{1}=\bar{g}_{i_{l}}^{2}\left(f_{0}\right) \in G_{i_{l}}^{1} \cap G_{i,}^{2},
$$

since the left-hand side belongs to $G_{i}^{1}$ while the right-hand side belongs to $G_{i}^{2}$. So by (2.5), using $g_{i}^{2}\left(f_{0}\right) \in \tilde{G}_{i}^{2}$, we get

$$
\begin{gathered}
\left\langle\bar{g}_{i_{l}}^{2}-g_{i_{l}}^{2}\left(f_{0}\right), g_{i_{l}}^{2}\left(f_{0}\right)\right\rangle=0, \\
\left\|g_{i_{i}}^{2}\left(f_{0}\right)\right\|^{2}=\left\langle\bar{g}_{i_{i}}^{2}, g_{i_{l}}^{2}\left(f_{0}\right)\right\rangle \leqslant \frac{1}{2}\left\|g_{i_{l}}^{2}\left(f_{0}\right)\right\|^{2}+\frac{1}{2}\left\|\bar{g}_{i_{l}}^{2}\right\|^{2},
\end{gathered}
$$

implying $\left\|g_{i_{1}}^{2}\left(f_{0}\right)\right\|^{2} \leqslant\left\|\bar{g}_{i_{1}}^{2}\right\|^{2}$. But the right-hand side is bounded by (2.4), contradicting (2.21). Hence the proof of the "only if" part is complete.

(if) For any $x \in G_{i}^{1}+G_{i}^{2}$, we have a unique representation

$$
x=g_{i}^{1}(x)+g_{i}^{2}(x), \quad\left(g_{i}^{1}(x), g_{i}^{2}(x)\right) \in G_{i}^{1} \times \tilde{G}_{i}^{2} .
$$

We define $P_{i}$ as in (2.13). Then for any $x \in G_{i}^{1}+G_{i}^{2}$, we have

$$
x=P_{i} x+\left(I-P_{i}\right) x
$$


So

$$
\begin{aligned}
\|x\|^{2} & =\langle x, x\rangle \geqslant\left\|P_{i} x\right\|^{2}+\left\|\left(I-P_{i}\right) x\right\|^{2}-2\left|\left\langle P_{i} x,\left(I-P_{i}\right) x\right\rangle\right| \\
& \geqslant\left\|P_{i} x\right\|^{2}+\left\|\left(I-P_{i}\right) x\right\|^{2}-2 \mu\left\|P_{i} x\right\|\left\|\left(I-P_{i}\right) x\right\| \\
& =\left(1-\mu^{2}\right)\left\|P_{i} x\right\|^{2}+\left(\mu\left\|P_{i} x\right\|-\left\|\left(I-P_{i}\right) x\right\|\right)^{2} \\
& \geqslant\left(1-\mu^{2}\right)\left\|P_{i} x\right\|^{2} .
\end{aligned}
$$

For any $f \in H$, we now let

$$
g_{i}^{1}(f) \equiv P_{i}\left(\mathbf{P}_{G_{i}^{1}+G_{i}^{2}} f\right) \in G_{i}^{1} .
$$

From (2.22), we get

$$
\left\|g_{i}^{1}(f)\right\| \leqslant \frac{1}{\left(1-\mu^{2}\right)^{1 / 2}}\left\|\mathbf{P}_{G_{i}^{1}+G_{i}^{2}} f\right\| \leqslant \frac{1}{\left(1-\mu^{2}\right)^{1 / 2}}\|f\| \quad \forall f \in H, \forall i \in \mathbf{Z}^{+},
$$

and

$$
\left\|g_{i}^{2}(f)\right\| \equiv\left\|\mathbf{P}_{G_{i}^{1}+G_{i}^{2}} f-g_{i}^{1}(f)\right\| \leqslant\left\|\mathbf{P}_{G_{i}^{1}+G_{i}^{2}} f\right\|+\left\|g_{i}^{1}(f)\right\| \leqslant\left[1+\frac{1}{\left(1-\mu^{2}\right)^{1 / 2}}\right]\|f\| .
$$

This proves (2.4)

COROLlaRY 9. Let $\left\{G_{i}^{1}\right\}_{i}$ and $\left\{G_{i}^{2}\right\}_{i}$ be two sequences of finite-dimensional subspaces in $H$ satisfying (2.1) and (2.2). If they satisfy

$$
G_{i}^{1} \cap G_{i}^{2}=\{0\} \quad \forall i \in \mathbf{Z}^{+},
$$

then

$$
\sup _{i} \cos \left(G_{i}^{1}, \tilde{G}_{i}^{2}\right)=1 \text {. }
$$

Therefore there exist some $f \in H$ such that (2.3) and (2.4) fail to hold.

Proof. Obvious.

Remark 10. A careful examination of the proof of Theorem 8 shows that (2.4) remains valid under the more general assumption that $\left\{G_{i}^{1}\right\},\left\{G_{i}^{2}\right\}$ are sequences of closed subspaces only, i.e., $\left\{G_{i}^{1}\right\},\left\{G_{i}^{2}\right\}$ need not be finite-dimensional.

Remark 11. According to von Neumann's alternating projection theorem [5], for any two closed subspaces $G^{1}, G^{2}$ in a Hilbert space $H$, one has

$$
s-\lim _{k \rightarrow \infty}\left(\mathbf{P}_{G^{1}} \mathbf{P}_{G^{2}}\right)^{k} x=\mathbf{P}_{G^{1} \cap G^{2}} x \quad \forall x \in H .
$$

Therefore, for any $f \in H$, its component in $G_{i}^{1} \cap G_{i}^{2}$ can be obtained iteratively as above.

3. Finite Element-Penalty Error Estimates. In Section 1, we have assumed that the family of products of finite-dimensional spaces $\left\{S_{h}^{1} \times S_{h}^{2} \subset H_{0 n}^{1} \times L_{m}^{2} \mid 0 \leqslant h \leqslant h_{0}\right\}$ is a continuous one parameter-family. In this section, we consider instead the simpler case, namely, we assume that we have a discrete one-parameter (sub-)family of finite-dimensional product spaces $\left\{S_{h_{i}}^{1} \times S_{h_{i}}^{2} \mid 0 \leqslant h_{i} \leqslant h_{0}, i \in \mathbf{Z}^{+}\right\}$with the following properties. 


$$
\begin{aligned}
& \begin{cases}\lim _{h_{i} \downarrow 0} \inf _{y_{h_{h}} \in S_{h_{i}}^{1}}\left\|y-y_{h_{i}}\right\|_{H_{0 n}^{1}}=0 & \forall y \in H_{0 n}^{1}, \\
\lim _{h_{i} \downarrow 0} \inf _{v_{h_{i}} \in S_{h_{i}}^{2}}\left\|v-v_{h_{i}}\right\|_{L_{m}^{2}}=0 & \forall v \in L_{m}^{2},\end{cases} \\
& S_{h_{i}}^{1} \subsetneq S_{h_{j}}^{1}, S_{h_{i}}^{2} \subsetneq S_{h_{j}}^{2} \quad \text { if } h_{i}>h_{j} .
\end{aligned}
$$

From $S_{h_{i}}^{1}$ and $S_{h_{i}}^{2}$, we construct $V_{h_{i}}^{1}$ and $V_{h_{i}}^{2}$ as in (1.18) and (1.19). In the sequel, we will denote $S_{h_{i}}^{1}, S_{h_{i}}^{2}, V_{h_{i}}^{1}$, and $V_{h_{i}}^{2}$ simply as $S_{i}^{1}, S_{i}^{2}, V_{i}^{1}$, and $V_{i}^{2}$, respectively.

Condition $(\mathrm{H})$. We say that the family $\left\{S_{i}^{1} \times S_{i}^{2}\right\}_{i \in \mathbf{Z}^{+}}$satisfies condition $(\mathrm{H})$ if the associated family $\left\{V_{i}^{1}, V_{i}^{2}\right\}_{i \in \mathbf{Z}^{+}}$satisfies

$$
\cos \left(V_{i}^{1}, \tilde{V}_{i}^{2}\right) \leqslant \mu<1 \quad \forall i \in \mathbf{Z}^{+},
$$

where $\tilde{V}_{i}^{2}=V_{i}^{2} \Theta\left(V_{i}^{1} \cap V_{i}^{2}\right)$ (cf. (2.5)).

A further auxiliary condition on $B$ will be needed. From now on we assume that $B$ and $\left\{S_{i}^{2}\right\}$ satisfy the following condition:

for any sequence $\left\{w_{i} \mid w_{i} \in V_{i}^{2}\right\}$ satisfying $\overline{\lim }_{i \rightarrow \infty}\left\|w_{i}\right\|<\infty$, there exists a sequence $\left\{v_{i} \mid v_{i} \in S_{i}^{2}\right\}$ such that $w_{i}=B v_{i}$ and $\overline{\lim }_{i \rightarrow \infty}\left\|v_{i}\right\|<\infty$.

It is easy to see that if $B$ is $1-1$, then (3.3) is valid for any $\left\{S_{i}^{2}\right\}$.

We are now in a position to prove the main theorem in this paper.

TheOREM 12 (MAIN ESTIMATES). Given a family of finite-dimensional subspaces $\left\{S_{i}^{1} \times S_{i}^{2} \mid i \in \mathbf{Z}^{+}\right\}$satisfying (3.1)-(3.3), let $\left\{\left(\hat{x}_{i}^{\varepsilon}, \hat{u}_{i}^{e}\right)\left(\equiv\left(\hat{x}_{h_{i}}^{e}, \hat{u}_{h_{i}}^{e}\right)\right) \mid i \in \mathbf{Z}^{+}\right\}$be the solutions of (1.16). Let $(0.3)$ and $(0.6)$ hold and let $(\hat{x}, \hat{u})$ be the solution of the optimal control problem (0.1) and (0.2). If condition $(\mathrm{H})$ is satisfied, then, for every $h_{i}>0$, $\varepsilon>0$, and $f \in L_{n}^{2}$, we have

$$
\begin{aligned}
& \left\|\hat{x}_{i}^{\varepsilon}-\hat{x}\right\|_{H_{0 n}^{1}}+\left\|\hat{u}_{i}^{\varepsilon}-\hat{u}\right\|_{L_{m}^{2}} \\
& \quad \leqslant C_{1}(\hat{x}, \hat{u}) \varepsilon+C_{2}\left[\inf _{y_{i} \in S_{i}^{1}}\left\|\hat{x}-y_{i}\right\|_{H_{0 n}^{1}}+\inf _{v_{i} \in S_{i}^{2}}\left\|\hat{u}-v_{i}\right\|_{L_{m}^{2}}\right],
\end{aligned}
$$

where $C_{1}(\hat{x}, \hat{u})$ depends on $\|(\hat{x}, \hat{u})\|$ (or equivalently, on $\left.\|f\|\right)$ with a linear growth rate and $C_{2}>0$ is a constant independent of $\hat{x}, \hat{u}, f, \varepsilon$, and $h_{i}$.

We first prove the following two lemmas.

LEMmA 13. Let the hypothesis of Theorem 12 hold. Let $\mathfrak{L}_{i} \equiv \mathfrak{L}_{h_{i}}$ be the mapping defined in (1.25), and $\mathcal{L}$ be as in (1.4). Then

$$
\mathfrak{L}_{i} f \rightarrow \mathfrak{L} f \quad \text { in } H_{0 n}^{1} \times L_{m}^{2} \text {, as } h_{i} \downarrow 0 \forall f \in L_{n}^{2}
$$

if and only if condition $(\mathrm{H})$ holds.

Proof. (if) Assume (H). By Theorem 8 there exists a sequence $\left\{\left(w_{i}^{1}, w_{i}^{2}\right) \in V_{i}^{1} \times\right.$ $\left.V_{i}^{2} \mid f_{i} \equiv \mathbf{P}_{V_{i}^{1}+V_{i}^{2}} f=w_{i}^{1}+w_{i}^{2}\right\}$ such that

$$
\varlimsup_{i \rightarrow \infty}\left[\left\|w_{i}^{1}\right\|+\left\|w_{i}^{2}\right\|\right]<\infty .
$$

Since $\mathfrak{L}_{i} f=\left(\hat{x}_{i}, \hat{u}_{i}\right),(1.28)$ gives

$$
f_{i}=\mathbf{P}_{V_{i}^{1}+V_{i}^{2}} f=\left(\dot{\hat{x}}_{i}-A \hat{x}_{i}\right)+\left(-B \hat{u}_{i}\right) \equiv \bar{w}_{i}^{1}+\bar{w}_{i}^{2},
$$


where $\left(\hat{x}_{i}, \hat{u}_{i}\right)$ is uniquely characterized by

$$
J\left(\hat{x}_{i}, \hat{u}_{i}\right)=\operatorname{Min}_{(x, u) \in S_{i}^{1} \times S_{i}^{2}} J(x, u) \quad \text { subject to } \dot{x}-A x-B u=f_{i}, x(0)=0 .
$$

Since $\left(w_{i}^{1}, w_{i}^{2}\right) \in V_{i}^{1} \times V_{i}^{2}$, there exists $\left(y_{i}, v_{i}\right) \in S_{i}^{1} \times S_{i}^{2}$ such that

$$
w_{i}^{1}=\dot{y}_{i}-A y_{i}, \quad w_{i}^{2}=B v_{i} .
$$

Therefore

$$
J\left(\hat{x}_{i}, \hat{u}_{i}\right) \leqslant J\left(y_{i}, v_{i}\right) .
$$

But, by (3.6) and (3.3), we can choose $v_{i}$ such that $\overline{\lim }\left\|v_{i}\right\|<\infty$, so

$$
\varlimsup_{i \rightarrow \infty} J\left(y_{i}, v_{i}\right) \leqslant C \varlimsup_{i \rightarrow \infty}\left\{\left\|w_{i}^{1}\right\|_{L_{n}^{2}}+\left\|v_{i}\right\|_{L_{n}^{2}}\right\}<\infty
$$

for some constant $C$ depending on $N_{1}, N_{2}$, and $M$ only. Therefore, from (3.7), $\left\{\left(\hat{x}_{i}, \hat{u}_{i}\right)\right\}$ has a subsequence converging weakly in $H_{0 n}^{1} \times L_{m}^{2}$ to some $(\bar{x}, \bar{u})$. Because of the lower semicontinuity of $J$ in $H_{0 n}^{1} \times L_{m}^{2}$, it is easy to see that this weak convergence is also strong, and the weak limit $(\bar{x}, \bar{u})$ satisfies

$$
\dot{\bar{x}}-A \bar{x}-B \bar{u}=\lim _{i \rightarrow \infty} f_{i}=f .
$$

Thus from uniqueness we see that $(\bar{x}, \bar{u})=(\hat{x}, \hat{u})$, the unique solution to the optimal control problem $(0.1)$ and $(0.2)$. Since every subsequence of $\left(\hat{x}_{i}, \hat{u}_{i}\right)$ converges to $(\hat{x}, \hat{u})$ strongly, we conclude

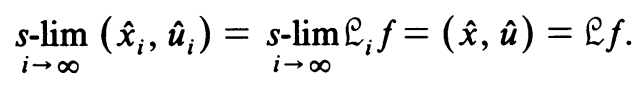

So (3.5) is proved.

(only if) If $\mathcal{L}_{i} f$ converges to $\varrho f$ for every $f$, we can choose

$$
w_{i}^{1} \equiv \dot{\hat{x}}_{i}-A \hat{x}_{i}, \quad w_{i}^{2} \equiv-B \hat{u}_{i},
$$

where $\left(\hat{x}_{i}, \hat{u}_{i}\right)=\mathscr{L}_{i} f$. Because $\left(\hat{x}_{i}, \hat{u}_{i}\right)=\mathscr{L}_{i} f$ converges to $(\hat{x}, \hat{u})=\mathscr{L} f$ strongly in $H_{0 n}^{1} \times L_{m}^{2}$, we have

$$
\lim _{i \rightarrow \infty}\left[\left\|w_{i}^{1}\right\|_{L_{n}^{2}}+\left\|w_{i}^{2}\right\|_{L_{n}^{2}}\right]=\|\dot{\hat{x}}-A \hat{x}\|_{L_{n}^{2}}+\|B \hat{u}\|_{L_{n}^{2}}<\infty
$$

proving (H).

Remark 14. By the uniform boundedness principle and Lemma 13, we conclude

$$
\sup _{i \in \mathbf{Z}^{+}}\left\{\left\|\mathcal{L}_{i}\right\|\right\}<\infty
$$

under condition $(\mathrm{H})$.

LEMMA 15. Assume the hypotheses of Theorem 12. Let $\left(\tilde{x}_{i}, \tilde{u}_{i}\right) \in S_{i}^{1} \times S_{i}^{2}$ satisfy

$$
a\left(\left[\begin{array}{c}
\hat{x}-\tilde{x}_{i} \\
\hat{u}-\tilde{u}_{i}
\end{array}\right],\left[\begin{array}{c}
y_{i} \\
v_{i}
\end{array}\right]\right)=0
$$

for all $\left(y_{i}, v_{i}\right) \in S_{i}^{1} \times S_{i}^{2}$. Then we have

$$
\left\|\tilde{x}_{i}-\hat{x}_{i}\right\|_{H_{0 n}^{1}}+\left\|\tilde{u}_{i}-\hat{u}_{i}\right\|_{L_{m}^{2}} \leqslant C\left\|\mathcal{L}_{i}\right\|\left[\inf _{y_{i} \in S_{i}^{1}}\left\|\hat{x}-y_{i}\right\|_{H_{0 n}^{1}}+\inf _{v_{i} \in S_{i}^{2}}\left\|\hat{u}-v_{i}\right\|_{L_{m}^{2}}\right]
$$

for some constant $C>0$ independent of $(\hat{x}, \hat{u})$. 
Proof. Let $\tilde{f}_{i}$ be defined by

$$
\tilde{f}_{i} \equiv \tilde{x}_{i}-A \tilde{x}_{i}-B \tilde{u}_{i}
$$

From (3.9) and [1] we have

(3.11) $\left\|\hat{x}-\tilde{x}_{i}\right\|_{H_{0 n}^{1}}+\left\|\hat{u}-\tilde{u}_{i}\right\|_{L_{m}^{2}} \leqslant C_{3}\left[\inf _{y_{i} \in S_{i}^{1}}\left\|\hat{x}-y_{i}\right\|_{H_{0 n}^{1}}+\inf _{v_{i} \in S_{i}^{2}}\left\|\hat{u}-v_{i}\right\|_{L_{m}^{2}}\right]$

for some constant $C_{3}$ depending on $N_{1}, N_{2}$, and $M$ only. Therefore, by (3.11)

$$
\begin{aligned}
\left\|f-\tilde{f}_{i}\right\|_{L_{n}^{2}} & =\left\|(\dot{\hat{x}}-A \hat{x}-B \hat{u})-\left(\tilde{x}_{i}-A \tilde{x}_{i}-B \tilde{u}_{i}\right)\right\|_{L_{n}^{2}} \\
& \leqslant\left\|\left(\dot{\hat{x}}-\hat{x}_{i}\right)-A\left(\hat{x}-\tilde{x}_{i}\right)\right\|_{L_{n}^{2}}+\left\|B\left(\hat{u}-\tilde{u}_{i}\right)\right\|_{L_{n}^{2}} \\
& \leqslant C_{4}\left[\inf _{y_{i} \in S_{i}^{1}}\left\|\hat{x}-y_{i}\right\|_{H_{0 n}^{1}}+\inf _{v_{i} \in S_{i}^{2}}\left\|\hat{u}-v_{i}\right\|_{L_{m}^{2}}\right]
\end{aligned}
$$

for some constant $C_{4}$ independent of $\hat{x}, \hat{u}, f, S_{i}^{1}$ and $S_{i}^{2}$.

From (1.14) and (3.9), we see that $\left(\tilde{x}_{i}, \tilde{u}_{i}\right)$ is characterized by the variational equation

$$
a\left(\left[\begin{array}{c}
\tilde{x}_{i} \\
\tilde{u}_{i}
\end{array}\right],\left[\begin{array}{c}
y_{i} \\
v_{i}
\end{array}\right]\right)+\left\langle\hat{p}, \dot{y}_{i}-A y_{i}-B v_{i}\right\rangle_{L_{n}^{2}}=0 \quad \forall\left(y_{i}, v_{i}\right) \in S_{i}^{1} \times S_{i}^{2} .
$$

Therefore $\left(\tilde{x}_{i}, \tilde{u}_{i}\right)$ is the unique solution in $S_{i}^{1} \times S_{i}^{2}$ of

$$
\left\{\begin{array}{l}
\underset{(x, u) \in S_{i} \times S_{i}^{2}}{\operatorname{Min}} J(x, u) \\
\text { subject to } \\
\dot{x}-A x-B u=\tilde{f}_{i}, \quad x(0)=0 .
\end{array}\right.
$$

Now, consider $\left(\tilde{x}_{i}, \tilde{u}_{i}\right)$. Let $f_{i} \equiv \mathbf{P}_{V_{i}^{1}+V_{i}^{2}} f$. We have

$$
\begin{aligned}
\left\|f-f_{i}\right\|_{L_{n}^{2}} & =\inf _{g_{i} \in V_{i}^{1}+V_{i}^{2}}\left\|f-g_{i}\right\|_{L_{n}^{2}} \\
& =\inf _{y_{i}, v_{i}}\left\|(\hat{\hat{x}}-A \hat{x}-B \hat{u})-\left(\dot{y}_{i}-A y_{i}-B v_{i}\right)\right\|_{L_{n}^{2}} \\
& \leqslant \inf _{y_{i}}\left\|\left[\left(\dot{\hat{x}}-\dot{y}_{i}\right)-A\left(\hat{x}-y_{i}\right)\right]\right\|_{L_{n}^{2}}+\inf _{v_{i}}\left\|B\left(\hat{u}-v_{i}\right)\right\|_{L_{n}^{2}} \\
& \leqslant C_{5}\left[\inf _{y_{i} \in S_{i}^{1}}\left\|\hat{x}-y_{i}\right\|_{H_{0 n}^{1}}+\inf _{v_{i} \in S_{i}^{2}}\left\|\hat{u}-v_{i}\right\|_{L_{m}^{2}}\right]
\end{aligned}
$$

for some constant $C_{5}$ independent of $\hat{x}, \hat{u}, f, S_{i}^{1}$ and $S_{i}^{2}$. Hence, (1.26), (3.13), (3.12), and (3.14) give

$$
\begin{aligned}
\left\|\tilde{x}_{i}-\hat{x}_{i}\right\|_{H_{0 n}^{1}}+\left\|\tilde{u}_{i}-\hat{u}_{i}\right\|_{L_{m}^{2}} & =\left\|\mathscr{L}_{i}\left(\tilde{f}_{i}\right)-\mathfrak{E}_{i}\left(f_{i}\right)\right\| \\
& \leqslant\left\|\mathfrak{L}_{i}\right\|\left\|\tilde{f}_{i}-f_{i}\right\|_{L_{n}^{2}} \leqslant\left\|\mathfrak{L}_{i}\right\|\left[\left\|\tilde{f}_{i}-f\right\|_{L_{n}^{2}}+\left\|f-f_{i}\right\|_{L_{n}^{2}}\right] \\
& \leqslant C_{6}\left\|\mathfrak{L}_{i}\right\|\left[\inf _{y_{i} \in S_{i}^{1}}\left\|\hat{x}-y_{i}\right\|_{H_{0 n}^{1}}+\inf _{v_{i} \in S_{i}^{2}}\left\|\hat{u}-v_{i}\right\|_{L_{m}^{2}}\right]
\end{aligned}
$$

with $C_{6} \equiv \max \left(C_{4}, C_{5}\right)$. Thus (3.10) is proved. 
We now give

Proof of Theorem 12. We use the triangle inequality

$$
\begin{aligned}
& \left\|\hat{x}_{i}^{\varepsilon}-\hat{x}\right\|_{H_{0 n}^{1}}+\left\|\hat{u}_{i}^{\varepsilon}-\hat{u}\right\|_{L_{m}^{2}} \\
& \leqslant \\
& \quad\left\|\hat{x}_{i}^{\varepsilon}-\hat{x}_{i}\right\|_{H_{0 n}^{1}}+\left\|\hat{x}_{i}-\tilde{x}_{i}\right\|_{H_{0 n}^{1}}+\left\|\tilde{x}_{i}-\hat{x}\right\|_{H_{0 n}^{1}}+\left\|\hat{u}_{i}^{\varepsilon}-\hat{u}_{i}\right\|_{L_{m}^{2}} \\
& \quad+\left\|\hat{u}_{i}-\tilde{u}_{i}\right\|_{L_{m}^{2}}+\left\|\tilde{u}_{i}-\hat{u}\right\|_{L_{m}^{2}} \equiv \sum_{i=1}^{6} T_{i} .
\end{aligned}
$$

By Corollary 6, we have

$$
T_{1}+T_{4} \leqslant \bar{K} \cdot\left\|\left(\hat{x}_{i}, \hat{u}_{i}\right)\right\| \cdot\left\|\mathscr{L}_{i}\right\|^{2} \cdot \varepsilon \quad \forall \varepsilon>0 .
$$

By Lemma 15, we have

$$
T_{2}+T_{5} \leqslant C_{7}\left\|\mathscr{L}_{i}\right\|\left[\inf _{y_{i} \in S_{i}^{1}}\left\|\hat{x}-y_{i}\right\|_{H_{0 n}^{1}}+\inf _{v_{i} \in S_{i}^{2}}\left\|\hat{u}-v_{i}\right\|_{L_{m}^{2}}\right]
$$

for some $C_{7}>0$.

By (3.11), we have

$$
T_{3}+T_{6} \leqslant C_{8}\left[\inf _{y_{i} \in S_{i}^{1}}\left\|\hat{x}-y_{i}\right\|_{H_{0 n}^{1}}+\inf _{v_{i} \in S_{i}^{1}}\left\|\hat{u}-v_{i}\right\|_{L_{m}^{2}}\right]
$$

for some $C_{8}>0$.

We define

$$
C_{1}(\hat{x}, \hat{u}) \equiv \sup _{i} \bar{K} \cdot\left\|\left(\hat{x}_{i}, \hat{u}_{i}\right)\right\| \cdot\left\|\mathscr{L}_{i}\right\|^{2} .
$$

By (3.8) and the strong convergence of $\left(\hat{x}_{i}, \hat{u}_{i}\right)$ to $(\hat{x}, \hat{u})$, we see that $C_{1}(\hat{x}, \hat{u})$ is finite, and it depends on $\|(\hat{x}, \hat{u})\|$ with a linear growth rate. We also define

$$
C_{2} \equiv C_{8}+C_{7} \cdot \sup _{i}\left\|\mathfrak{L}_{i}\right\| \text {. }
$$

Then $C_{2}<\infty$ by (3.8).

Combining (3.15)-(3.20), we conclude (3.4).

Remark 16. One might try to prove Lemma 13 (and Theorem 12) from the boundedness of the multiplier $\hat{p}_{h}$ in (1.29). Actually, our argument above shows that if condition (H) is not satisfied, then not all multipliers $\hat{p}_{h}$ are bounded as $h \downarrow 0$.

As in [1], we say that $S_{h} \subset H_{l}^{s}$ is an $(r, s)$-system if for all $v \in H_{l}^{k}(0, T)$, there exists $v_{h} \in S_{h}$ such that

$$
\left\|v-v_{h}\right\|_{H_{i}^{\eta}} \leqslant C h^{\mu}\left\|_{v}\right\|_{H^{++\eta}} \quad \forall 0 \leqslant \eta \leqslant \min \{k, s\}, \eta \in \mathbf{N},
$$

where $\mu=\min \{r-\eta, k-\eta\}$ and $C$ is independent of $h$ and $v$.

Corollary 17. Let $\left(\hat{x}_{i}^{\varepsilon}, \hat{u}_{i}^{\varepsilon}\right)$ solve (1.16). Let $(0.3)$ and $(0.6)$ hold and $(\hat{x}, \hat{u})$ solve (0.1) and (0.2) with $\hat{x} \in H_{n}^{l_{1}}$ and $\hat{u} \in H_{m}^{l_{2}}$. Let $S_{i}^{1} \subseteq H_{0 n}^{1}$ be an $\left(r_{1}, 1\right)$-system and let $S_{i}^{2} \subseteq L_{m}^{2}$ be an $\left(r_{2}, 0\right)$-system such that $(3.3)$ and condition $(\mathrm{H})$ are satisfied. Then for each $h_{i}>0, f \in L_{n}^{2}$, and $\varepsilon>0$, there exist constants $C_{1}(\hat{x}, \hat{u})$ (depending on $\left.\|(\hat{x}, \hat{u})\|\right)$ and $C_{2}$ such that

$$
\left\|\hat{x}_{i}^{\varepsilon}-\hat{x}\right\|_{H_{0 n}^{1}}+\left\|\hat{u}_{i}^{\varepsilon}-\hat{u}\right\|_{L_{m}^{2}} \leqslant C_{1}(\hat{x}, \hat{u}) \varepsilon+C_{2}\left[h_{i}^{\mu_{1}}\|\hat{x}\|_{H_{n}^{\mu_{1}+1}}+h_{i}^{\mu^{2}}\|\hat{u}\|_{H_{m}^{\mu_{2}^{2}}}\right]
$$

where $\mu_{1}=\min \left(r_{1}-1, l_{1}-1\right)$ and $\mu_{2}=\min \left(r_{2}, l_{2}\right)$. 
From (3.21) we see that optimal error bounds are obtained when $\mu_{1}=\mu_{2}$ and $\varepsilon=O\left(h_{i}^{\mu_{1}}\right)$. Therefore, if $(\hat{x}, \hat{u}) \in\left(H_{n}^{l+1} \cap H_{0 n}^{l}\right) \oplus H_{m}^{l}$ for some $l \in \mathbf{Z}^{+}$, we usually choose

$$
r_{1}-1=r_{2} \text {. }
$$

Condition $(\mathrm{H})$ is a very strong assumption. If it is not satisfied, one can show that sharp error estimates like (3.4) are impossible.

THEOREM 18. Let (0.3) and (0.6) hold, and let $\left\{S_{h}^{1} \times S_{h}^{2}\right\}_{h}$ be a discrete (or continuous) one-parameter family of closed subspaces of $H_{0 n}^{1} \times L_{m}^{2}$ satisfying (3.1)-(3.3) (or (1.15)). If $(\mathrm{H})$ is not satisfied, then there cannot exist nonnegative error estimation functions $E_{1}(\varepsilon, \hat{x}, \hat{u})$ and $E_{2}(h, \hat{x}, \hat{u})$ satisfying

$$
\lim _{\varepsilon \downarrow 0} E_{1}(\varepsilon, \hat{x}, \hat{u})=0, \quad \lim _{h \downarrow 0} E_{2}(h, \hat{x}, \hat{u})=0
$$

such that

$$
\left\|\hat{x}_{h}^{\varepsilon}-\hat{x}\right\|_{H_{0 n}^{1}}+\left\|\hat{u}_{h}^{\varepsilon}-\hat{u}\right\|_{L_{m}^{2}} \leqslant E_{1}(\varepsilon, \hat{x}, \hat{u})+E_{2}(h, \hat{x}, \hat{u}) .
$$

Proof. Assume the contrary that (3.23) and (3.24) hold. By Corollary 6, we have

$$
\begin{aligned}
\left\|\hat{x}_{h}-\hat{x}\right\|_{H_{0 n}^{1}}+\left\|\hat{u}_{h}-\hat{u}\right\|_{L_{m}^{2}} & =\lim _{\varepsilon \downarrow 0}\left[\left\|\hat{x}_{h}^{\varepsilon}-\hat{x}\right\|_{H_{0 n}^{1}}+\left\|\hat{u}_{h}^{\varepsilon}-\hat{u}\right\|_{L_{m}^{2}}\right] \\
& \leqslant \lim _{\varepsilon \downarrow 0}\left[E_{1}(\varepsilon, \hat{x}, \hat{u})+E_{2}(h, \hat{x}, \hat{u})\right]=E_{2}(h, \hat{x}, \hat{u}) .
\end{aligned}
$$

Hence

$$
\lim _{h \downarrow 0}\left[\left\|\hat{x}_{h}-\hat{x}\right\|_{H_{0 n}^{1}}+\left\|\hat{u}_{h}-\hat{u}\right\|_{L_{m}^{2}}\right] \leqslant \lim _{h \downarrow 0} E_{2}(h, \hat{x}, \hat{u})=0 .
$$

Let

$$
w_{h}^{1} \equiv \dot{\hat{x}}_{h}-A \hat{x}_{h} \in V_{h}^{1}, \quad w_{h}^{2} \equiv-B \hat{u}_{h} \in V_{h}^{2} .
$$

For any $f \in L_{n}^{2},(1.26)$ gives

$$
\mathbf{P}_{V_{h}^{1}+V_{h}^{2}} f=w_{h}^{1}+w_{h}^{2},
$$

and (3.25) gives

$$
\lim _{h \downarrow 0}\left[\left\|w_{h}^{1}\right\|_{L_{n}^{2}}^{2}+\left\|w_{h}^{2}\right\|_{L_{n}^{2}}^{2}\right]=\|\dot{\hat{x}}-A \hat{x}\|_{L_{n}^{2}}^{2}+\|B \hat{u}\|_{L_{n}^{2}}^{2}<\infty .
$$

This means that $(\mathrm{H})$ is satisfied, a contradiction.

From the proofs of Theorems 12 and 18, we conclude

CoROllaRY 19. Let (0.3), (0.6), and (3.1)-(3.3) hold. Let $\left(\hat{x}_{i}^{\varepsilon}, \hat{u}_{i}^{\varepsilon}\right)$ and $(\hat{x}, \hat{u})$ denote, respectively, the solutions of (1.16) and (0.1), (0.2), and let $\mathcal{L}_{i}$ be defined as in (1.25). Then the following conditions are equivalent:

(1) Condition $(\mathrm{H})$.

(2) $\sup _{i \in \mathbf{Z}^{+}}\left\{\left\|\mathcal{L}_{i}\right\|\right\}<\infty$.

(3) There exist $C_{1}(\hat{x}, \hat{u})>0$ depending on $(\hat{x}, \hat{u})$ only and $C_{2}>0$ independent of $\hat{x}$, $\hat{u}, f, h_{i}$, and $\varepsilon$ such that, for all $h_{i}>0$ and $\varepsilon>0$,

$$
\left\|\hat{x}_{i}^{\varepsilon}-\hat{x}\right\|_{H_{0 n}^{1}}+\left\|\hat{u}_{i}^{\varepsilon}-\hat{u}\right\|_{L_{m}^{2}} \leqslant C_{1}(\hat{x}, \hat{u}) \varepsilon+C_{2}\left[\inf _{y \in S_{i}^{1}}\|\hat{x}-y\|_{H_{0 n}^{1}}+\inf _{v \in S_{i}^{2}}\|\hat{u}-v\|_{L_{m}^{2}}\right] .
$$


(4) There exist two error estimation functions $E_{1}(\varepsilon, \hat{x}, \hat{u})$ and $E_{2}\left(h_{i}, \hat{x}, \hat{u}\right)$ such that

$$
\lim _{\varepsilon \downarrow 0} E_{1}(\varepsilon, \hat{x}, \hat{u})=0, \quad \lim _{h_{i} \downarrow 0} E_{2}\left(h_{i}, \hat{x}, \hat{u}\right)=0,
$$

and

$$
\left\|\hat{x}_{i}^{\varepsilon}-\hat{x}\right\|_{H_{0 n}^{1}}+\left\|\hat{u}_{i}^{\varepsilon}-\hat{u}\right\|_{L_{m}^{2}} \leqslant E_{1}(\varepsilon, \hat{x}, \hat{u})+E_{2}\left(h_{i}, \hat{x}, \hat{u}\right) .
$$

Theorem 18 tells us that, without $(\mathrm{H}), \varepsilon$ and $h$ appearing in error estimates must be coupled in general. The following gives one of the simplest type of such errors.

THEOREM 20. Let $(0.3)$ and $(0.6)$ hold. Let $\left(\hat{x}_{\varepsilon}, \hat{u}_{\varepsilon}\right)$ solve $(1.1)$ and $(\hat{x}, \hat{u})$ solve $(0.1)$, (0.2). Assume that $\left(\hat{x}_{\varepsilon}, \hat{u}_{\varepsilon}\right)$ converges to $(\hat{x}, \hat{u})$ in $\left(H_{n}^{l+1} \cap H_{0 n}^{1}\right) \oplus H_{m}^{l}$ for some $l \in \mathbf{Z}^{+}$. Let $S_{h}^{1} \subseteq H_{0 n}^{1}$ and $S_{h}^{2} \subseteq L_{m}^{2}$ be $\left(r_{1}, 1\right)$ - and $\left(r_{2}, 0\right)$-systems, respectively. Then there exist constants $C_{1}>0$ and $C_{2}>0$ (both depending on $\left.(\hat{x}, \hat{u})\right)$ such that, for $\varepsilon>0$ sufficiently small,

$$
\left\|\hat{x}_{h}^{\varepsilon}-\hat{x}\right\|_{H_{0 n}^{1}}+\left\|\hat{u}_{h}^{\varepsilon}-\hat{u}\right\|_{L_{m}^{2}} \leqslant C_{1} \varepsilon+\frac{C_{2} h^{\mu}}{\sqrt{\varepsilon}}\left[\|\hat{x}\|_{H_{n}^{\mu+1}}+\|\hat{u}\|_{H_{m}^{\mu}}\right],
$$

where $\mu=\min \left(l-1, r_{1}-1, r_{2}\right)$.

Proof. We use the triangle inequality

$$
\begin{aligned}
& \left\|\hat{x}_{h}^{\varepsilon}-\hat{x}\right\|_{H_{0 n}^{1}}+\left\|\hat{u}_{h}^{\varepsilon}-\hat{u}\right\|_{L_{m}^{2}} \\
& \quad \leqslant\left\|\hat{x}_{h}^{\varepsilon}-\hat{x}_{\varepsilon}\right\|_{H_{0 n}^{1}}+\left\|\hat{x}_{\varepsilon}-\hat{x}\right\|_{H_{0 n}^{1}}+\left\|\hat{u}_{h}^{\varepsilon}-\hat{u}_{\varepsilon}\right\|_{L_{m}^{2}}+\left\|\hat{u}_{\varepsilon}-\hat{u}\right\|_{L_{m}^{2}} .
\end{aligned}
$$

From Theorem 2, we have

$$
\left\|\hat{x}_{\varepsilon}-\hat{x}\right\|_{H_{0 n}^{1}}+\left\|\hat{u}_{\varepsilon}-\hat{u}\right\|_{L_{m}^{2}} \leqslant C_{1} \varepsilon \quad \forall \varepsilon>0,
$$

for some constant $C_{1}$ depending on $(\hat{x}, \hat{u})$.

We use the bilinear form $a_{\varepsilon}(\cdot, \cdot)$ in (1.13). It satisfies

$$
\left|a_{\varepsilon}\left(\left[\begin{array}{l}
x_{1} \\
u_{1}
\end{array}\right],\left[\begin{array}{l}
x_{2} \\
u_{2}
\end{array}\right]\right)\right| \leqslant \frac{\bar{C}_{2}}{\varepsilon}\left\|\left(x_{1}, u_{1}\right)\right\|_{H_{0 n}^{1} \times L_{m}^{2}}\left\|\left(x_{2}, u_{2}\right)\right\|_{H_{0 n}^{1} \times L_{m}^{2}}
$$

for all $\left(x_{1}, u_{1}\right),\left(x_{2}, u_{2}\right) \in H_{0 n}^{1} \times L_{m}^{2}$. Since $\left(\hat{x}_{h}^{\varepsilon}, \hat{u}_{h}^{\varepsilon}\right),\left(\hat{x}_{\varepsilon}, \hat{u}_{\varepsilon}\right)$ satisfy the variational equation

$$
a_{\varepsilon}\left(\left[\begin{array}{c}
\hat{x}_{\varepsilon}-\hat{x}_{h}^{\varepsilon} \\
\hat{u}_{\varepsilon}-\hat{u}_{h}^{\varepsilon}
\end{array}\right],\left[\begin{array}{c}
y_{h} \\
v_{h}
\end{array}\right]\right)=0 \quad \forall\left(y_{h}, v_{h}\right) \in S_{h}^{1} \times S_{h}^{2},
$$

it follows from [1] that

$$
\begin{aligned}
\left\|\hat{x}_{\varepsilon}-\hat{x}_{h}^{\varepsilon}\right\|_{H_{0 n}^{1}}+\left\|\hat{u}_{\varepsilon}-\hat{u}_{h}^{\varepsilon}\right\|_{L_{m}^{2}} & \\
\leqslant & \left(\frac{\bar{C}_{3}}{\varepsilon}\right)^{1 / 2}\left[\inf _{y_{h} \in S_{h}^{1}}\left\|\hat{x}_{\varepsilon}-y_{h}\right\|_{H_{0 n}^{1}}+\inf _{v_{h} \in S_{h}^{2}}\left\|\hat{u}_{\varepsilon}-v_{h}\right\|_{L_{m}^{2}}\right] \\
& \leqslant \frac{\bar{C}_{4}^{1 / 2}}{\sqrt{\varepsilon}}\left[h^{\mu_{1}}\left\|\hat{x}_{\varepsilon}\right\|_{H_{n}^{l+1}}+h^{\mu_{2}}\left\|\hat{u}_{\varepsilon}\right\|_{H_{m}^{\prime}}\right] .
\end{aligned}
$$

Letting $\mu=\min \left(\mu_{1}, \mu_{2}\right)$, choosing $C_{2}$ properly from $\bar{C}_{4}^{1 / 2}$, and combining (3.27)(3.29), we get (3.26). 
4. Applications. We apply the theorems in Section 3 to several types of problem (0.1), (0.2) and its penalized finite element approximation (1.16). We produce a class of problems and approximating subspaces for which condition $(\mathrm{H})$ (and Theorem 12) holds, and specific examples for which it does not.

The approximating subspaces we consider are piecewise polynomial spaces. Let $0=t_{0}<t_{1}<\cdots<t_{N}=T$ be a uniform parition of $[0, T]$ with mesh length $h=$ $T / N=t_{i+1}-t_{i}$. Let $S_{h}^{(r, s)}=\left\{p \in C^{s}[0, T]: p\right.$ is a polynomial of degree $r-1$ on each subinterval $\left.\left[t_{i}, t_{i+1}\right], i=0, \ldots, N-1\right\}$. In the approximation (1.17), $S_{h}^{1}$ and $S_{h}^{2}$ will be the $(r, s)$-systems of $n$-fold and $m$-fold products of subspaces of $S_{h}^{(r, s)}$, respectively. If locally supported $B$-spline bases for $S_{h}^{(r, s)}$ are used, then the matrix equation resulting from (1.17) will have the symmetric block banded structure

$$
\left[\begin{array}{c:c}
A_{n n} & A_{n m} \\
\left.\hdashline A_{m n}\right\lrcorner & A_{m m}
\end{array}\right]
$$

where each

$$
A_{k l}=\left[\begin{array}{cccc}
B_{11} & B_{12} & \cdots & B_{1 l} \\
B_{21} & B_{22} & & \vdots \\
\vdots & & \ddots & \\
B_{k 1} & \cdots & & B_{k l}
\end{array}\right]
$$

and $B_{i j}$ are banded matrices according to the choice of $S_{h}^{(r, s)}$.

Example 1. Consider the optimal control problem

$$
\left\{\begin{array}{l}
\dot{x}(t)=B u(t)+f(t), \quad 0 \leqslant t \leqslant T, f \in L_{n}^{2}(0, T), B=\text { a constant } n \times m \text { matrix, } \\
x(0)=0 \in \mathbf{R}^{n}, \\
\underset{(x, u) \in H_{0 n}^{1} \times L_{m}^{2}}{\operatorname{Min}} J(x, u)=\int_{0}^{T}\left[\left\langle\dot{x}, N_{1} \dot{x}\right\rangle+\left\langle x, N_{2} x\right\rangle+\langle u, M u\rangle\right] d t .
\end{array}\right.
$$

We let $S_{h}^{1}=\Pi_{i=1}^{n}\left\{\varphi \in S_{h}^{(k+1,1)} \mid \varphi(0)=0\right\}$ and $S_{h}^{2}=\prod_{i=1}^{m} S_{h}^{(k, 0)}$. All hypotheses of Theorem 12 are evident except condition $(\mathrm{H})$. To show this let $V_{h}^{1}=\left\{\dot{\varphi} \mid \varphi \in S_{h}^{1}\right\}$, $V_{h}^{2}=\left\{B \varphi \mid \varphi \in S_{h}^{2}\right\}$. We claim $V_{h}^{1} \cap V_{h}^{2}=V_{h}^{2}$. Then $\tilde{V}_{h}^{2}=V_{h}^{2} \ominus\left(V_{h}^{1} \cap V_{h}^{2}\right)=$ $\{0\}$, and $\mu=\cos \left(V_{h}^{1}, \tilde{V}_{h}^{2}\right)=0$ giving condition $(\mathrm{H})$.

For each vector-valued function $\bar{\psi} \in V_{h}^{2}$, any of its components is a scalar function $\psi$ which can be represented piecewise as

$$
\psi=\sum_{j=0}^{k-1} a_{i j}\left(t-t_{i}\right)^{j}, \quad t_{i} \leqslant t \leqslant t_{i+1}, i=0,1, \ldots, N-1,
$$

and

$$
\psi\left(t_{i}^{+}\right)=\psi\left(t_{i}^{-}\right) .
$$

We wish to find a vector-valued function $\bar{\varphi} \in S_{h}^{1}$ such that $\dot{\bar{\varphi}}=\bar{\psi}$.

Any component $\varphi$ of $\bar{\varphi}$ satisfies

$$
\begin{gathered}
\varphi=\sum_{j=0}^{k} b_{i j}\left(t-t_{i}\right)^{j}, \quad t_{i} \leqslant t \leqslant t_{i+1}, i=0, \ldots, N-1, \\
\varphi\left(t_{0}\right)=\varphi(0)=0, \\
\varphi\left(t_{i}^{-}\right)=\varphi\left(t_{i}^{+}\right), \quad i=1, \ldots, N-1,
\end{gathered}
$$




$$
\varphi^{\prime}\left(t_{i}^{-}\right)=\varphi^{\prime}\left(t_{i}^{+}\right), \quad i=1, \ldots, N-1 .
$$

From (4.4), (4.5), and (4.6), we get, respectively,

$$
\begin{aligned}
b_{0,0} & =0, \\
\sum_{j=0}^{k} b_{i j} h^{j} & =b_{i+1,0}, \quad i=0, \ldots, N-2, \\
\sum_{j=1}^{k} j b_{i j} h^{j-1} & =b_{i+1,1}, \quad i=0, \ldots, N-2 .
\end{aligned}
$$

In order that $\dot{\varphi}=\psi$, we must have

$$
\sum_{j=1}^{k} j b_{i j}\left(t-t_{i}\right)^{j-1}=\sum_{j=0}^{k-1} a_{i j}\left(t-t_{i}\right)^{j}, \quad t_{i} \leqslant t \leqslant t_{i+1}
$$

or

$$
\sum_{j=0}^{k-1}(j+1) b_{i, j+1}\left(t-t_{i}\right)^{j}=\sum_{j=0}^{k-1} a_{i j}\left(t-t_{i}\right)^{j} .
$$

From the linear independence of polynomials over each subinterval, we must have

$$
(j+1) b_{i, j+1}=a_{i j}, \quad j=0, \ldots, k-1, i=0, \ldots, N-2 .
$$

Substituting (4.10) into (4.8) and (4.9) gives

$$
\begin{gathered}
b_{i+1,0}=b_{i, 0}+\sum_{j=1}^{k} \frac{1}{j} a_{i, j-1} h^{j}, \quad i=0, \ldots, N-2, \\
\sum_{j=1}^{k} a_{i, j-1} h^{j-1}=a_{i+1,0}, \quad i=0, \ldots, N-2 .
\end{gathered}
$$

Note that in (4.11), the expression can be also made valid even for $i=0$ by choosing

$$
\varphi(t)=\int_{0}^{t} \psi(\tau) d \tau, \quad t \in\left[0, t_{1}\right] .
$$

Relations (4.7), (4.10) and (4.11) (with (4.13)) define $b$ 's in terms of $a$ 's. Relation (4.12) is automatically satisfied because it is just (4.3). Therefore $b$ 's can be determined from $a$ 's in a unique way.

Therefore for any $\bar{\psi} \in V_{h}^{2}$ there exists a $\bar{\varphi} \in V_{h}^{1}$ such that $\dot{\bar{\varphi}}=\bar{\psi}$. That is, $V_{h}^{2} \subset V_{h}^{1}$. Hence Theorem 12, Corollary 17 and estimate (3.21) hold with $\mu_{1}=\mu_{2}=$ $k$.

Computational solutions of (1.17) were obtained for the specific problem

$$
\begin{gathered}
\left\{\begin{array}{l}
\dot{x}=u+\cos t, \quad 0 \leqslant t \leqslant 1, \\
x(0)=0,
\end{array}\right. \\
\underset{(x, u) \in H_{01}^{1} \times L_{1}^{2}}{\int_{0}^{1}\left[\dot{x}^{2}(t)+u^{2}(t)\right] d t,},
\end{gathered}
$$

with $S_{h}^{1}=\left\{\varphi \in S_{h}^{(4,1)}: \varphi(0)=0\right\}, S_{h}^{2}=S_{h}^{(3,0)}$ ( $C^{1}$-cubics and $C^{0}$-quadratics) [12]. The dimension of both $S_{h}^{1}$ and $S_{h}^{2}$ is $2 N+1$ and hence the matrix (4.1) is of order $4 N+2$.

Table 1 and Figure 1 give the errors between $\left(\hat{x}_{h}^{\varepsilon}, \hat{u}_{h}^{\varepsilon}\right)$ and the exact solution $(\hat{x}(t), \hat{u}(t))=\left(\frac{1}{2} \sin t,-\frac{1}{2} \cos t\right)$ for various $h$ and $\varepsilon$. Note that the slopes obtained in Figure 1 indicate the sharpness of Corollary 17. 
TABLE 1

$\left\|\hat{x}_{h}^{\varepsilon}-\hat{x}\right\|_{H^{1}}+\left\|\hat{u}_{h}^{\varepsilon}-\hat{u}\right\|_{L^{2}}$ error for Example 1, (4.14), (4.15)

\begin{tabular}{|c|c|c|c|c|}
\hline $\mathrm{n}$ & $10^{-2}$ & $10^{-4}$ & $10^{-5}$ & $10^{-6}$ \\
\hline$\frac{1}{2}$ & $.461 \times 10^{-2}$ & $.263 \times 10^{-3}$ & $.259 \times 10^{-3}$ & $.252 \times 10^{-3}$ \\
\hline$\frac{1}{4}$ & $.461 \times 10^{-2}$ & $.607 \times 10^{-4}$ & $.394 \times 10^{-4}$ & $.350 \times 10^{-4}$ \\
\hline$\frac{1}{8}$ & $.461 \times 10^{-2}$ & $.466 \times 10^{-4}$ & $.475 \times 10^{-5}$ & $.472 \times 10^{-5}$ \\
\hline$\frac{1}{16}$ & $.461 \times 10^{-2}$ & $.463 \times 10^{-4}$ & $.468 \times 10^{-5}$ & $.612 \times 10^{-6}$ \\
\hline
\end{tabular}

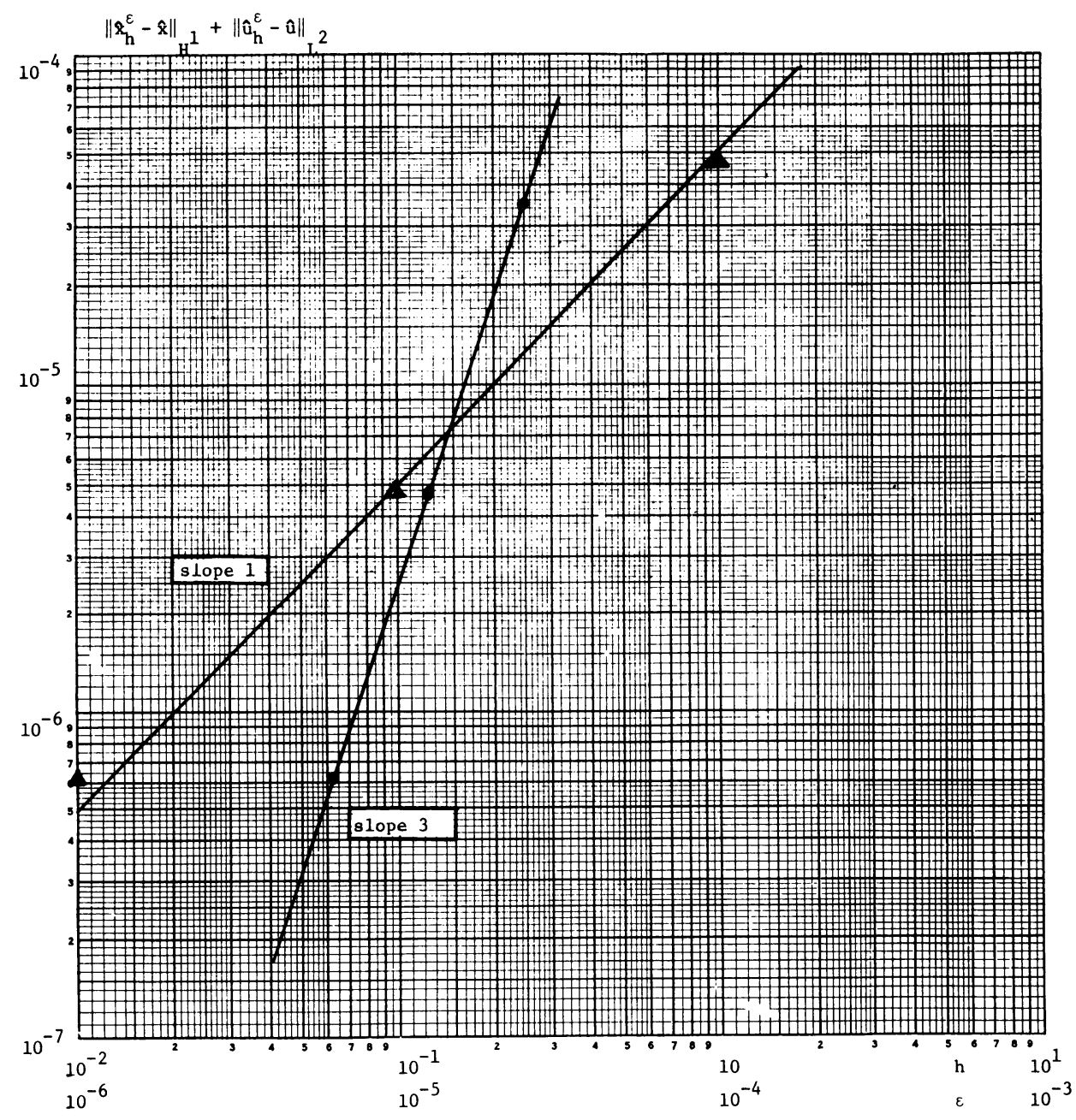

FIGURE 1

Example 1 error vs $h\left(\varepsilon=10^{-6}\right) \circ \circ \circ$

Example l error vs $\varepsilon(h=1 / 16) \triangle \triangle \triangle$ 
Example 2. Consider the same optimal control problem as in Example 1, with the matrix $B$ invertible $(m=n)$. But, we now choose

$$
S_{h}^{1}=\prod_{i=1}^{n}\left\{\varphi \in S_{h}^{(k+1,0)} \mid \varphi(0)=0\right\}, \quad S_{h}^{2}=\prod_{i=1}^{n} S_{h}^{(k+1,0)}, \quad k \in \mathbf{Z}^{+}, k>1 .
$$

In this case,

$$
V_{h}^{1}=\left\{\dot{\varphi} \mid \varphi \in S_{h}^{1}\right\}, \quad V_{h}^{2}=S_{h}^{2} .
$$

Arguing in the same manner as in Example 1, we can verify that

$$
V_{h}^{1} \cap V_{h}^{2}=\prod_{i=1}^{n} S_{h}^{(k, 0)}
$$

thus $\tilde{V}_{h}^{2} \neq\{0\}$. In order to be able to apply the theory in Section 3, we must find $\tilde{V}_{h}^{2}$, the orthogonal complement of $V_{h}^{1} \cap V_{h}^{2}$ in $V_{h}^{2}$. Unfortunately, there does not seem to be any simple representation for basis elements in $\tilde{V}_{h}^{2}$. This points out a general difficulty in applying the theory of Section 3. If there exist convenient representations (e.g., as piecewise polynomials) for basis elements in $\tilde{V}_{h}^{2}$, then $\cos \left(V_{h}^{1}, \tilde{V}_{h}^{2}\right)$ can be computed by optimization techniques.

Example 3. Let the optimal control problem be

$$
\left\{\begin{array}{l}
\dot{x}(t)=(1+\cos t) x(t)+u(t)+f(t), \quad 0 \leqslant t \leqslant T, f \in L_{n}^{2}(0, T), \\
x(0)=0 \in \mathbf{R}^{n} \\
\underset{(x, u)}{\operatorname{Min}} J(x, u)=\int_{0}\left[\left\langle\dot{x}, N_{1} \dot{x}\right\rangle+\left\langle x, N_{2} x\right\rangle+\langle u, M u\rangle\right] d t .
\end{array}\right.
$$

Let

$$
S_{h}^{1} \equiv S_{h}^{\left(k_{1}, j_{1}\right)} \cap H_{0 n}^{1}, \quad S_{h}^{2} \equiv S_{h}^{\left(k_{2}, j_{2}\right)}, \quad k_{1}, k_{2}, j_{1}, j_{2} \in \mathbf{N}, k_{1}, k_{2}>1
$$

Then

$$
V_{h}^{1}=\left\{\dot{\varphi}-(1+\cos t) \varphi \mid \varphi \in S_{h}^{1}\right\}, \quad V_{h}^{2}=S_{h}^{2} .
$$

It is a simple exercise to verify that $V_{h}^{1} \cap V_{h}^{2}=\{0\}$. Therefore Corollary 9 holds. In this case, no asymptotic sharp error estimate like (3.4) is possible.

If the governing equation is changed to

$$
\dot{x}(t)=x(t)+(2+\cos t) \cdot u(t)+f(t),
$$

for example, the same conclusion also holds.

Example 4. Let the control system dynamics be autonomous:

$$
\dot{x}(t)=A x(t)+B u(t)+f(t),
$$

where $A$ and $B$ are constant matrices, and let $S_{h}^{1}, S_{h}^{2}$ be chosen as in (4.16). Consider $V_{h}^{1} \cap V_{h}^{2}$. If $\varphi_{h} \in S_{h}^{1}$ and $\psi_{h} \in S_{h}^{2}$ satisfies

$$
\dot{\varphi}_{h}-A \varphi_{h}=B \psi_{h} \in V_{h}^{1} \cap V_{h}^{2}, \quad \varphi_{h}(0)=0,
$$

then

$$
\varphi_{h}(t)=\int_{0}^{t} e^{-A(t-s)} \psi_{h}(s) d s .
$$

If the constant matrix $A$ has only simple eigenvalues which are nonzero, then all the entries of $\exp (A t)$ consist of (scalar) linear combinations of exponential functions 
$\sum c_{i} e^{\lambda_{i} t}\left(\lambda_{i}=\right.$ an eigenvalue). We see that (4.17) and (4.18) can hold only for $\varphi_{h}$ and $\psi_{h}$ which are constant vectors. Hence the dimension of $V_{h}^{1} \cap V_{h}^{2}$ cannot exceed a bounded integer. It is not hard to see that condition $(\mathrm{H})$ fails.

If $A$ has zero eigenvalues with high multiplicity, then some entries of $\exp (A t)$ are polynomials. Thus, the dimensions of $V_{h}^{1} \cap V_{h}^{2}$ may increase somewhat, but in general are still bounded by a fixed integer. Hence condition $(\mathrm{H})$ fails.

In fact, Examples 3 and 4 indicate that except in very special circumstances (i.e., special choices of $A$ and $B$ ) such as Example 1, sharp error estimates do not hold and $\varepsilon, h$ must be coupled.

Acknowledgement. We wish to thank F. Deutsch and W. Hager for motivating discussions.

Department of Mathematics

Pennsylvania State University

University Park, Pennsylvania 16802

Department of Mathematics

Pennsylvania State University

University Park, Pennsylvania 16802

Department of Mathematics

Sichuan University

Chengdu, Sichuan

People's Republic of China

Martin-Marietta Company

Denver, Colorado 80200

1. I. BABUSKA \& A. K. AzIz, The Mathematical Foundations of the Finite Element Method with Applications to Partial Differential Equations (A. K. Aziz, ed.), Academic Press, New York, 1972.

2. A. V. Balakrishnan, "On a new computing technique in optimal control," SIAM J. Control., v. 6, 1968, pp. 149-173.

3. W. E. Bosarge \& O. G. Johnson, "Error bounds of high order accuracy for the state regulator problem via piecewise polynomial approximation," SIAM J. Control, v. 9, 1971, pp. 15-28.

4. G. Chen \& W. H. Mills, "Finite elements and terminal penalization for quadratic cost optimal control problems governed by ordinary differential equations," SIAM J. Control Optim., v. 19, 1981, pp. 744-764.

5. F. DeutsCH, “The alternating method of von-Neumann," in Multivariate Approximation Theory (W. Schempp and K. Zeller, eds.), Birkhäuser Verlag, Basel, 1979.

6. R. S. FALK, "A finite element method for the stationary Stokes equations using trial functions which do not have to satisfy div $v=0$," Math. Comp., v. 30, 1976, pp. 698-702.

7. R. S. FAlK \& J. T. KING, "A penalty and extrapolation method for the stationary Stokes equation," SIAM J. Numer. Anal., v. 13, 1976, pp. 814-829.

8. I. C. GOHBERG \& M. G. KREIN, Introduction to the Theory of Linear Nonselfadjoint Operators, Transl. Math. Monographs, vol. 18, Amer. Math. Soc., Providence, R. I., 1969.

9. J. L. Lions, Optimal Control of Systems Governed by Partial Differential Equations, Springer-Verlag, Berlin and New York, 1971.

10. B. T. PolyaK, "The convergence rate of the penalty function method," Zh. Vychisl. Mat. $i$ Mat. Fiz., v. 11, 1971, pp. 3-11. (Russian)

11. D. L. RuSsell, Mathematics of Finite Dimensional Control Systems, Theory and Design, Marcel Dekker, New York, 1979.

12. G. Strang \& G. Fix, An Analysis of the Finite Element Method, Prentice-Hall, Englewood Cliff, N. J., 1973. 